\title{
CONNECTIONS AND GENERALIZED GAUGE TRANSFORMATIONS
}

\author{
Simon Davis \\ Institut für Mathematik \\ Universität Potsdam \\ D-14415, Potsdam, Germany
}

\begin{abstract}
The derivation of the standard model from a higher-dimensional action suggests a further study of the fibre bundle formulation of gauge theories to determine the variations in the choice of structure group that are allowed in this geometrical setting. The action of transformations on the projection of fibres to their submanifolds are characteristic of theories with fewer gauge vector bosons, and specific examples are given, which may have phenomenological relevance. The spinor space for the three generations of fermions in the standard model is described algebraically.
\end{abstract}

Keywords: bundles, connections, fibre coordinates, parallelizable spheres, division algebras, force unification

AMS Subject Classification: 81T13, 81T30, 81V22 


\section{Introduction}

It has been shown recently that supertstring theory possesses improved finiteness properties at each order [1][2][3] and at large orders in the series expansion for scattering amplitudes [4], suggesting that a consistent quantum theory containing general relativity in the low-energy limit has been obtained. Phenomenonologically relevant models of elementary particle interactions also might be included for a suitable choice of superstring vacuum [5][6]. A principle for selecting a specific superstring vacuum would be needed as a theoretical basis for any choice of ground state. One possibility, motivated by topology change arising in the path integral for quantum gravity [7], would involve an integral over different background configurations, dominated by a sum over solutions to the string equations of motion, with the geometries weighted by a string field theory action [8][9]. Alternatively, one might wish to consider a principle directly constraining geometries associated with a Kaluza-Klein unification of general relativity with the elementary particle interactions.

The attempt to unify the elementary particle interactions with gravity has gradually led to the development of theories which may be regarded as generalizations of standard gauge theories [8][10][11][12]. As a mathematical structure, a category of fibre bundles larger than that of the principal bundles may be considered. For example, the fibre could be taken to be any $n$-dimensional complete simply connected manifold, which would be diffeomorphic to $S^{n}$ if specific bounds are imposed on the sectional curvature [13][14]. Given that the space- and time-oriented manifolds $(M, g)$ admitting spinor structures are parallelizable [15], the feasibility of projecting vector fields and one-forms in a parallelizable total space to the base manifold would depend on the parallelizability of the fibre. Choosing the fibre to be an $n$-sphere, this condition would imply that $n=1,3$ or 7 , which, together with the topological equivalence of a locally trivial fibre bundle with total space $S^{2 n-1}$ and $S^{n-1}$ with the classical Hopf fibration when $n=1,2,4$, and 8 [16], and the differentiable equivalence when $n=1,2$ and 4, implies a link between the division algebras and a restricted category of bundles defined by theoretical constraints necessary for the construction of generalized gauge theories. In a previous investigation [17], a physical requirement imposed on the transformation rule of the connection form of a general bundle led to the constraint of the fibre admitting a global parallelism or equivalently independence of the commutation relations of the tangent vector fields with respect to the fibre space coordinates. This is particularly relevant to an approach to force unification utilizing a link between the internal symmetries and the different division algebras [18]-[22], relating the number of types of elementary particles with the number of division algebras [23]-[26].

\section{Gauge Theories, the Seven-Sphere and the Division Algebras}

All Kaluza-Klein theories unifying gravity with the elementary particle forces describe matter fields which are transforming under the isometry group of the compact manifold defining the extra dimensions so that the octonionic algebra cannot arise as a the symmetry algebra of the action. In earlier attempts to construct an octonionic gauge theory [27][28][29], there was no invariance under gauge transformations of the potential taking values only in the base space. The reasons for the lack of a gauge principle can be understood by considering the fibre-coordinate dependence of the connection form transformation rule for $S^{7}$ bundles.

The potential is defined to be the coefficient of the coordinate differentials $\left\{d x^{\mu}\right\}$ in the expression for the pull-back of the connection one-form on the base manifold from a section of a principal 
bundle.

$$
\begin{aligned}
A_{\mu} & =\omega\left(\sigma_{*} \cdot \xi_{\mu}\right)=\left(\sigma^{*} \omega\right)\left(\xi_{\mu}\right) \\
\xi_{\mu} & =\frac{\partial}{\partial x^{\mu}} \\
\sigma^{*} & =A_{\mu} d x^{\mu}
\end{aligned}
$$

Similarly, the field strength defines the components of the curvature two-form $\Omega=d \omega+\omega \wedge \omega$ and $\Omega=F_{\mu \nu} d x^{\mu} \wedge d x^{\nu}$.

The pure Yang-Mills action $\int_{M} d^{4} x \operatorname{Tr}\left(F \wedge F^{*}\right)$ is bounded by the functional, $\int_{M} d^{4} x \operatorname{Tr}(F \wedge F)$, given by the second Chern class for a principal bundle in the expansion

$$
\begin{aligned}
c(\Omega) & =\operatorname{Det}\left(I+\frac{i}{2 \pi} \Omega\right)=1+c_{1}(\Omega)+c_{2}(\Omega)+\ldots \\
c_{0} & =1 \\
c_{1} & =\frac{i}{2 \pi} \operatorname{Tr} \Omega \\
c_{2} & =\frac{1}{8 \pi^{2}}[\operatorname{Tr} \Omega \wedge \Omega-\operatorname{Tr} \Omega \wedge \operatorname{Tr} \Omega]
\end{aligned}
$$

where $\Omega$ is the curvature form. While the second Chern class may be generalized to the manifold $S^{7}$, the following theorem shows that a similar generalization of the Yang-Mills functional will not be invariant under gauge transformations of potentials taking values in the tangent space at a chosen origin of $S^{7}$ as the structure constants of the octonion algebra $f^{a b c}$ do not satisfy the Jacobi identity preventing closure.

Specifically, invariance of the Lagrangian $L\left(A_{\mu}^{k}, \partial A_{\mu}^{k}\right)$ with respect to the infinitesimal variation $\delta A_{\mu}^{k}=\partial_{\mu} \epsilon^{k}+f^{k}{ }_{l m} \epsilon^{l} A_{\mu}^{m}[30]$ requires

$$
\begin{aligned}
\frac{\partial L}{\partial\left(\partial_{\mu} A_{\nu}^{k}\right)}+\frac{\partial L}{\partial\left(\partial_{\nu} A_{\mu}^{k}\right)} & =0 \\
\frac{\partial L}{\partial A_{\nu}^{l}}+\frac{\partial L}{\partial\left(\partial_{\nu} A_{\mu}^{k}\right)} f_{k l m} A_{\mu}^{m} & =0 \\
\frac{\partial L}{\partial A_{\mu}^{k}} f_{k l m} A_{\mu}^{k}+\frac{\partial L}{\partial\left(\partial_{\nu} A_{\mu}^{k}\right)} f_{k l m} \partial_{\nu} A_{\mu}^{m} & =0
\end{aligned}
$$

While the first two conditions together imply that $L=L\left(F_{\mu \nu}^{k}\right)$, using $\frac{\partial L}{\partial A_{\mu}^{k}}=2 \frac{\partial L}{\partial F_{\mu \nu}^{k}} f_{k l m} A_{\nu}^{m}$, whereas the last relation is equivalent to

$$
\begin{gathered}
2 \frac{\partial L}{\partial F_{\mu \nu}^{l}} f_{k l m} A_{\nu}^{m} f_{n p k} A_{\mu}^{p}-2 \frac{\partial L}{\partial F_{\mu \nu}^{k}} f_{n m k} \partial_{\nu} A_{\mu}^{k}=0 \\
\frac{\partial L}{\partial F_{\mu \nu}^{l}}\left(f_{k l m} f_{k n p}+f_{k l p} f_{k m n}\right) A_{\mu}^{p} A_{\nu}^{m}-\frac{\partial L}{\partial F_{\mu \nu}^{k}} f_{k n m}\left(\partial_{\nu} A_{\mu}^{k}-\partial_{\mu} A_{\nu}^{k}\right)= \\
\frac{\partial L}{\partial F_{\mu \nu}^{k}} f_{k n m} F_{\mu \nu}^{m}+\frac{\partial L}{\partial F_{\mu \nu}^{l}}\left(f_{k l m} f_{k n p}+f_{k l p} f_{k m n}+f_{k l n} f_{k p m}\right) A_{\mu}^{p} A_{\nu}^{m}=0
\end{gathered}
$$


While the quadratic term containing the gauge potential vanishes for a Lie group by the Jacobi identity, it would equal $\frac{\partial L}{\partial F_{\mu \nu}^{l}} \varphi_{m l n p} A_{\mu}^{p} A_{\nu}^{m}$ when the structure constants of the octonions are used, where $\varphi_{m l n p}$ represents the non-associativity of the algebra, $\left[e_{\alpha}, e_{\beta}, e_{\gamma}\right]=\left(e_{\alpha} e_{\beta}\right) e_{\gamma}-e_{\alpha}\left(e_{\beta} e_{\gamma}\right) \equiv$ $2 \varphi_{\alpha \beta \gamma \delta} e_{\delta}$. While an additional term of the form $\varphi_{k l m n} A_{\mu}^{l} A^{\nu m} \partial_{\nu} \epsilon^{n}$ or $\varphi_{k l m n} \partial_{\nu} A_{\mu}^{l} A^{\nu m} \epsilon^{n}$ could be included in the transformation rule for $A_{\mu}^{k}$, and the definition of the field strength could be altered so that it contains $\varphi_{k l m n}$, it is not feasible to cancel $\frac{\partial L}{\partial F_{\mu \nu}^{l}} \phi_{m l n p} A_{\mu}^{p} A_{\nu}^{m}$ based on the tensor structure of the equations of motion.

An octonionic gauge theory has been proposed using a bimodule representation of the octonion algebra [31]. Recalling that the octonion algebra is defined by the multiplication relations

$$
\begin{aligned}
e_{0}^{2} & =e_{0} \quad e_{0} e_{i}=e_{i} e_{0}=e_{i} \\
e_{i} e_{j} & =-\delta_{i j} e_{0}+\epsilon_{i j k} e_{k} \\
\epsilon_{i j k} & =1 \text { for }(i j k)=(123),(145),(167),(264),(257),(347),(356)
\end{aligned}
$$

using the conventions of [31], which can be summarized as $e_{\mu} e_{\nu}=C_{\mu \nu}^{\sigma} e_{\sigma}, \mu, \nu, \sigma=0,1, \ldots, 7$. The left representation is $\left(L_{\mu}\right)_{\sigma \nu}=\left(C_{\mu}\right)_{\nu}^{\sigma} \equiv C_{\mu \nu}^{\sigma}$ and the right representation is $\left(R_{\mu}\right)_{\sigma \nu}=\left(\tilde{C}_{\mu}\right)_{\nu}^{\sigma} \equiv C_{\nu \mu}^{\sigma}$ with $L_{0}=R_{0}=I d$. Then $\lambda_{i}=i \frac{L_{i}}{4}$ and $\rho_{j}=i \frac{R_{j}}{4}$ satisfy the commutation relations

$$
\begin{aligned}
& {\left[\lambda_{i}, \lambda_{j}\right]=\frac{i}{2} \epsilon_{i j k} \lambda_{k}+2\left[\rho_{j}, \lambda_{i}\right]} \\
& {\left[\rho_{i}, \rho_{j}\right]=-\frac{i}{2} \epsilon_{i j k} \rho_{k}+2\left[\lambda_{j}, \rho_{i}\right]}
\end{aligned}
$$

and the trace relations

$$
\operatorname{Tr}\left(\lambda_{i} \lambda_{j}\right)=\operatorname{Tr}\left(\rho_{i} \rho_{j}\right)=\frac{1}{2} \delta_{i j}
$$

Suppose a gauge potential is defined to be $A_{\mu}(x)=A_{\mu}^{i}(x) \lambda_{i}$, leading to the covariant derivative $\mathcal{D}_{\mu}=\partial_{\mu}+i g A_{\mu}$. Under a gauge transformation corresponding to the element $\Omega(x)=e^{i \alpha^{i}(x) \lambda_{i}}$, $\mathcal{D}_{\mu} \psi(x) \rightarrow e^{i \alpha^{i} \lambda_{i}} \mathcal{D}_{\mu} \psi(x)$ for any fermion field $\psi(x)$, and $\left[\mathcal{D}_{\mu}, \mathcal{D}_{\nu}\right]$ also transforms covariantly, allowing one to immediately formulate an invariant $-\frac{1}{2} \operatorname{Tr}\left(F_{\mu \nu} F^{\mu \nu}\right)$.

However, the gauge tranformation which leaves this action invariant

$$
\begin{aligned}
A_{\mu}(x) \rightarrow A_{\mu}^{\prime}(x) & =\Omega(x) A_{\mu}(x) \Omega^{-1}(x)+\frac{i}{g}\left(\partial_{\mu} \Omega(x)\right) \Omega^{-1}(x) \\
& =e^{i \alpha \cdot \lambda} A_{\mu} e^{-i \alpha \cdot \lambda}-\frac{1}{g} \partial_{\mu} \alpha^{i} \lambda_{i} \\
& =A_{\mu}+\left[i \alpha \cdot \lambda, A_{\mu}\right]-\frac{1}{2}\left[\alpha \cdot \lambda,\left[\alpha \cdot \lambda, A_{\mu}\right]\right]+\ldots-\frac{1}{g} \partial_{\mu} \alpha^{i} \lambda_{i}
\end{aligned}
$$

contains not only the generators $\lambda_{i}$ associated with the left representation of the octonion algebra but also generators arising from multiple commutators of $\lambda_{i}$ and $\rho_{j}$. To linear order in $\alpha$,

$$
\begin{aligned}
& A_{\mu}^{\prime}=A_{\mu}^{i i} \lambda_{i}+2 i \alpha^{j} A_{\mu}^{k}\left[\rho_{k}, \lambda_{j}\right] \\
& A_{\mu}^{i \prime}=A_{\mu}^{i}-\frac{1}{2} \epsilon_{i j k} \alpha^{j} A_{\mu}^{k}-\frac{1}{g} \partial_{\mu} \alpha^{i}
\end{aligned}
$$


and

$$
\begin{aligned}
F_{\mu \nu}^{\prime}= & F_{\mu \nu}^{i} \lambda_{i}-2 g \alpha^{k}\left(A_{\mu}^{\prime i} A_{\nu}^{\prime j}-A_{\nu}^{\prime i} A_{\mu}^{\prime j}\right)\left[\lambda_{i},\left[\rho_{k}, \lambda_{j}\right]\right] \\
& +2 i\left[\partial_{\mu}\left(\alpha^{j} A_{\nu}^{\prime k}\right)-\partial_{\nu}\left(\alpha^{j} A_{\nu}^{\prime k}\right)\right]\left[\rho_{k}, \lambda_{j}\right] \\
F_{\mu \nu}^{\prime i}= & \partial_{\mu} A_{\nu}^{\prime i}-\partial_{\nu} A_{\mu}^{\prime i}-\frac{1}{2} g \epsilon_{i j k} A_{\mu}^{\prime j} A_{\nu}^{\prime k}
\end{aligned}
$$

The trace relations

$$
\begin{aligned}
\operatorname{Tr}\left(\tau_{i}\left[\rho_{j}, \lambda_{k}\right]\right) & =\frac{i}{8} \epsilon_{i j k} \\
\operatorname{Tr}\left(\left[\lambda_{i}, \rho_{j}\right]\left[\lambda_{k}, \rho_{l}\right]\right) & =\frac{-1}{32}\left[\epsilon_{i j k l}+2\left(\delta_{i k} \delta_{j l}-\delta_{i l} \delta_{j k}\right)\right]
\end{aligned}
$$

can be used to express the Lagrangian in component form

$$
\mathcal{L}=-\frac{1}{4}\left(\partial_{\mu} A_{\nu}^{i}-\partial_{\nu} A_{\mu}^{i}\right)\left(\partial^{\mu} A^{\nu i}-\partial^{\nu} A^{\mu i}\right)-\frac{1}{16} g^{2} A_{\mu}^{j} A_{\nu}^{k}\left(A^{j \mu} A^{k \nu}-A^{k \mu} A^{j \nu}\right)
$$

but the action is not invariant under the substitution $A_{\mu}^{i} \rightarrow A_{\mu}^{i}$ as (2.11) becomes

$$
\begin{aligned}
& -\frac{1}{4}\left(\partial_{\mu} A_{\nu}^{i}-\partial_{\nu} A_{\mu}^{i}\right)\left(\partial^{\mu} A^{\nu i}-\partial^{\nu} A^{\mu i}\right)-\frac{1}{16} g^{2} A_{\mu}^{j} A_{\nu}^{k}\left(A^{j \mu} A^{k \nu}-A^{k \mu} A^{j \nu}\right) \\
& +\frac{1}{2}\left[\partial_{\mu}\left(\epsilon_{i j k} \alpha^{j} A_{\nu}^{k}+\frac{1}{g} \partial_{\nu} \alpha^{i}\right)-\partial_{\nu}\left(\epsilon_{i j k} \alpha^{j} A_{\mu}^{k}+\frac{1}{g} \partial_{\mu} \alpha^{i}\right)\right] \cdot \partial^{\mu} A^{\nu i} \\
& +\frac{1}{8} g^{2}\left(A_{\mu}^{j} A_{\nu}^{k}-A_{\nu}^{j} A_{\mu}^{k}\right)\left(\frac{1}{2} \epsilon_{p q}^{j} \alpha^{p} A_{\mu}^{q}+\frac{1}{g} \partial^{\mu} \alpha^{j}\right) \cdot A_{k}^{\nu}
\end{aligned}
$$

and the extra terms in (2.13) are not total derivatives. Consequently, the transformation of the potential $A_{\mu} \rightarrow A_{\mu}^{\prime}$ is required and this involves generators other than those corresponding to the left representation of the octonion algebra.

Writing $A_{\mu}^{\prime}=A_{\mu}^{\prime A B} J_{A B}$, a transformation $A_{\mu}^{\prime} \rightarrow A_{\mu}=\Omega^{-1} A_{\mu} \Omega-\frac{i}{g} \Omega^{-1}\left(\partial_{\mu} \Omega\right), \Omega \in G_{2}$ can be found such that $A_{\mu}$ has only non-zero components $A_{\mu}^{i}$ multiplying the generators $\lambda_{i}$. From (2.10), $F_{\mu \nu}=F_{\mu \nu}^{A B} J_{A B}$ has non-zero components corresponding to the generators of $G_{2}$ and the Lagrangian $-\frac{1}{2} \operatorname{Tr}\left(F_{\mu \nu} F^{\mu \nu}\right)$ actually should be invariant under the entire group of $G_{2}$ transformations. This symmetry is broken only when one specializes to a particular choice for the vanishing components of the gauge potential.

This result may also be understood from the context of non-associative deformations of gauge theories [32]. These generalizations are based on an algebraic structure consisting of $\mathrm{M}$ set of generators $\left\{T_{p i}\right\}, p=1, \ldots, M$. Together, the entire set of generators form an associative Lie algebra structure. Restriction to one set of generators, $T_{i}^{p}$, $\mathrm{p}$ fixed, leads to a problem with closure of the algebra.

$$
\left[T_{i}^{p}, T_{j}^{p}\right]=f_{i j k}^{p} T_{k}^{p}+\sum_{n=1}^{M} \sigma_{n}^{p}\left[T_{j}^{n}, T_{i}^{p}\right]
$$

The deviation from associativity can be measured by the associator

$$
\begin{aligned}
J\left(T_{i}^{p}, T_{j}^{p}, T_{k}^{p}\right) & =\epsilon^{i j k}\left(T_{i}^{p}, T_{j}^{p}, T_{k}^{p}\right)=\epsilon^{i j k}\left[\left(T_{i}^{p} T_{j}^{p}\right) T_{k}^{p}-T_{i}^{p}\left(T_{j}^{p} T_{k}^{p}\right)\right] \\
& =\sigma_{n}^{p}\left(\left[T_{i}^{p},\left[T_{k}^{n}, T_{j}^{n}\right]\right]+\left[T_{j}^{p},\left[T_{i}^{n}, T_{k}^{p}\right]\right]+\left[T_{k}^{p},\left[T_{j}^{n}, T_{i}^{p}\right]\right]\right)
\end{aligned}
$$


The lack of closure of the algebra associated with a single set of generators, or equivalently, the coupling of the gauge potentials corresponding to distinct sets of generators, leads to a Lagrangian, based on only the field strengths $F_{\mu \nu}^{p}$, which contains extra nonlinear terms. Therefore, when the algebra $T_{i}^{p}, p$ fixed represents the octonions, the entire algebra is given by the matrices associated with left and right multiplication introduced earlier. The theory obtained is therefore a special case of this general procedure of deforming gauge theories, where the Lagrangian is part of an action with the larger $G_{2}$ symmetry generated by the combined set of fourteen generators.

A general procedure has been developed for constructing a non-associative gauge theory, where the potential takes values in a non-associative algebra A [33]. The gauge symmetry of the action, however, is the automorphism group of $\mathrm{A}, G_{A}$, so that given a symmetric, bi-linear nondegenerate form $\langle u \mid v\rangle, u, v \in A$, satisfying the invariance condition $\langle g u \mid g v\rangle$, a Lagrangian $L_{0}=\frac{1}{4}\left\langle F_{\mu \nu} \mid F_{\mu \nu}\right\rangle$ may be constructed. If $\Lambda_{p}$ is the set of A-valued p-forms, then it is necessary to assume that $g \in g l\left(\Lambda_{1}\right), \xi \in \Lambda_{1}$ and

$$
\begin{aligned}
& \text { (i) } g(\omega \omega)=(g \omega)(g \omega) \\
& \text { (ii) } d \xi+\xi \xi=0 \\
& \text { (iii) } d(g \omega)=g(d \omega)-\xi(g \omega)-(g \omega) \xi
\end{aligned}
$$

Although it is not known if there is a solution for $g$ and $\xi$ for any given non-associative algebra, it can be assumed that one exists when $\mathrm{A}$ is the octonion algebra and the symmetry group $G_{A}$ is then $G_{2}$.

A common property of all of these theories is that the action possesses a Lie group symmetry even though it has been constructed so that it seems to include only components in the nonassociative octonion algebra. This provides further support for the assertion proven in [17] and Appendix A, which implies that a pure gauge theory with a symmetry defined only by the nonassociative algebra does not exist. In addition, it may be noted that although $\left(g^{-1} A_{\mu}\right) g=g^{-1}\left(A_{\mu} g\right)$ for alternative algebras by Artin's theorem,

$$
\left\{g^{-1}\left(A_{\mu} g\right)\right\}\left\{\left(g^{-1} A_{\nu}\right) g\right\} \neq g^{-1}\left(A_{\mu} A_{\nu}\right) g
$$

in general for non-associative algebras, so that the validity of invariance under finite gauge transformations as a consequence of invariance under infinitesimal transformations cannot be proven for non-associative algebras [33]. Since an extensive investigation of the possibility of constructing a pure gauge theory based only on the non-associative algebra has revealed that Lie group structure is essential, this property will be assumed in the following sections, although the seven-sphere shall continue to be used in this geometrical approach to the internal symmetry spaces.

A connection between the gauge groups in the standard model and the division algebras has been established using Clifford algebras, since a tensor product of division algebras defines the spinor space $T=\mathbb{C} \otimes \mathbb{H} \otimes \mathbb{O}[34]$, and there is an algebraic procedure for obtaining gauge symmetries from the left action algebra for this spinor space. The adjoint algebra is $T_{L} \sim R_{0,9}$, where $R_{p, q}$ is the Clifford algebra of the pseudo-Euclidean space $R^{p, q}$, and $T_{L}(2) \sim \mathbb{C}(32)$, the complexification of $R_{1,9}$, the equivalent of the Dirac algebra in ten-dimensional Minkowksi space-time.

The subspace of 2-vectors of $R_{p, q}$ closes under commutation and it is isomorphic to the Lie algebra $s o(p, q)$. Thus, the two-vector basis $\left\{e_{L p q}, p, q=1, \ldots, 6, p \neq q\right\}$ of $\mathbb{O}_{L} \sim R_{0,6}$ as 15 dimensional and isomorphic to $s o(6) \sim s u(4)$. The intersection of $\mathrm{su}(4)$ with $L G_{2}=\left\{e_{L a b}-e_{L c d}\right.$ : 
$\left.e_{a} e_{b}=e_{c} e_{d}\right\}$ is $s u(3)=\left\{e_{L p q}-e_{L r s}: e_{p} e_{q}=e_{r} e_{s}, p, q, r, s \neq 7\right\}$. The $\mathrm{SU}(3)$ gauge symmetry of the strong interactions arises as part of the $\mathrm{SO}(1,9)$ Lorentz transformations and not as an internal symmetry in ten dimensions. This is consistent with the use of string theory to describe the strong interactions and gravity.

Beginning with a ten-dimensional Lagrangian

$$
\begin{aligned}
\mathcal{L}_{1,9} & =\mathcal{L}_{\text {gauge }}+\mathcal{L}_{\phi}+\mathcal{L}_{1,9}^{\text {ferm }} \\
\mathcal{L}_{1,9}^{\text {ferm }} & =\left\langle\Psi \mid \partial_{1,9} \Psi\right\rangle
\end{aligned}
$$

and $\mathcal{L}_{\text {gauge }}$ is a ten-dimensional action for the spin-one gauge field. After using the projector distinguishing between matter and anti-matter multiplets, $R_{1,9}$ is projected to $R_{1,3} \otimes S O(6)$, the bosonic part of the action is based on the covariant derivative appropriate for $R_{1,3} \otimes S U(4)$. The SU(4) symmetry must then be broken to SU(3) to reproduce the QCD action, while the $S U(2) \times U(1)$ symmetry, arising from $R_{1,3}$ is present in the Weinberg-Salam model. While the scalar Lagrangian $\mathcal{L}_{\phi}$ can be used to generate spontaneous symmetry breaking, it is not necessary for the reduction of the gauge group from $\mathrm{SU}(4)$ to $\mathrm{SU}(3)$. The fermion Lagrangian may be reduced to the standard lepton-quark Lagrangian in four dimensions. Using the projector $\rho_{ \pm}=\frac{\left(1 \pm i e_{L 7}\right)}{2}$, one may re-express the fermion term as

$$
\begin{aligned}
\left\langle\rho_{+} \Psi \mid \not \partial 1_{1,3}\left(\rho_{+} \Psi\right)\right\rangle & +\left\langle\rho_{-} \Psi \mid \not \partial_{1,3}\left(\rho_{-} \Psi\right)\right\rangle \\
& \left\langle\rho_{+} \Psi \mid \partial_{0,6}\left(\rho_{-} \Psi\right)\right\rangle+\left\langle\rho_{-} \Psi \mid \partial_{0,6}\left(\rho_{+} \Psi\right)\right\rangle
\end{aligned}
$$

The last two terms represent matter/anti-matter transitions that are not observed, and they vanishg upon imposing the conditions

$$
\partial_{0,6}\left(\rho_{ \pm} \Psi\right)=0
$$

The solutions to these constraints have a dependency on the coordinates in the extra six dimensions which gives rise to the $\mathrm{SU}(2)$ and $\mathrm{SU}(3)$ symmetries of the standard model. Thus, the fermionic part of the theory may be derived by using a decomposition of modules of the Clifford algebra $R_{1,9}$ based on idempotents. This decomposition is unique by the Krull-Schmidt theorem [35], since they are direct sums of irreducible modules.

As the spinor space $\mathrm{T}$ is 64-dimensional, it is just large enough to describe a family consisting of a lepton doublet and a quark doublet with three distinct colours and the corresponding antifamily. Three generations of leptons can be obtained by choosing a preferred octonionic unit and three quarternionic subalgebras of $\mathbb{O}[36]$, although a theoretical basis for the three generations of quarks or the values of the isospin and hypercharge for fermions within each generation has yet to be found using this technique. Alternatively, the spinor space $\oplus_{i=1}^{3} \mathbb{C}_{i} \otimes \mathbb{H}_{i} \otimes \mathbb{O}_{i}$ can be used to include the three generations of fermions.

\section{Constraints on Gauge Transformations acting on Bundles with $S^{7}$ Fibres and Their Sub-bundles}

Gauge transformations may be viewed as active transformations on the standard fibre. Let $\sigma(x)=y \in G, \sigma^{\prime}(x)=\sigma(x) \cdot g(x)=y \cdot g \in G$. If $\xi \in T_{x}(M)$,

$$
\begin{aligned}
\sigma_{*}^{\prime} \cdot \xi & =R_{g *} \sigma_{*} \cdot \xi+L_{y *} g_{*} \cdot \xi \\
& =R_{g *} \sigma_{*} \cdot \xi+L_{(y \cdot g) *} L_{g^{-1 *}}\left(g_{*} \cdot \xi\right)
\end{aligned}
$$


Given a connection form

$$
\begin{aligned}
\omega\left(\sigma_{*}^{\prime} \cdot \xi\right)=L_{(y \cdot g) *}^{-1} \mathcal{V}\left(\sigma_{*}^{\prime} \cdot \xi\right) & =L_{(y \cdot g) *}^{-1}\left[\mathcal{V}\left(R_{g_{*}} \sigma_{*} \cdot \xi\right)+\mathcal{V}\left(L_{(y \cdot g) *} L_{g^{-1} *} g_{*} \cdot \xi\right)\right] \\
& =L_{(y \cdot g) *}^{-1} R_{g *} \mathcal{V}\left(\sigma_{*} \cdot \xi\right)+L_{g^{-1} *} g_{*} \cdot \xi
\end{aligned}
$$

where $\mathcal{V}$ represents the projection onto the vertical subspace $V_{(x, \sigma(x))}(E) \sim V_{\sigma(x)}(E)$. Since $\omega\left(\sigma_{*} \cdot \xi\right)=L_{y *}^{-1} \mathcal{V}\left(\sigma_{*} \cdot \xi\right)$

$$
\begin{aligned}
\omega\left(\sigma_{*}^{\prime} \cdot \xi\right) & =L_{(y \cdot g) *}^{-1} R_{g *} L_{y *} \omega\left(\sigma_{*} \cdot \xi\right)+L_{g^{-1} *} g_{*} \cdot \xi \\
& =a d\left(g^{-1}\right) \omega\left(\sigma_{*} \cdot \xi\right)+L_{g^{-1} *} g_{*} \cdot \xi
\end{aligned}
$$

Setting $\xi$ equal to $\partial_{\mu}$, one recovers the standard gauge transformation law

$$
A_{\mu}^{\prime}=\operatorname{ad}\left(g^{-1}\right) A_{\mu}+g^{-1} \partial_{\mu} g
$$

The dependence on $y$ in equation (3.2) has disappeared, which is necessary if the theory is to be formulated in the four-dimensional space-time M.

If the fibre is $S^{7}$, it admits a parallelism associated with the existence of octonions as an 8-dimensional division algebra over the real numbers. Consider the action of $\mathrm{SO}(8)$ by right multiplication on a trivial $S^{7}$ bundle. Suppose $L_{g^{-1}{ }_{*}} g_{*} \cdot \xi_{\mu}$ is an arbitrary element of the 28-dimensional Lie algebra of $\mathrm{SO}(8)$. Let $\iota_{L}$ an embedding of octonions in $\mathrm{SO}(8)$, so that left multiplication by $\mathrm{y}$ is represented by right multiplication by the matrix $\iota_{L}(y)$. Then, independence with respect to the fibre coordinate of the inhomogeneous term in the gauge transformation implies that $\lambda_{y *} \cdot L_{g^{-1} *} \cdot\left(g_{*} \cdot \xi\right) \cdot\left(\iota_{L}\left(y^{-1}\right)^{T}\right)_{*}$, or equivalently that the row vector $y \cdot\left(d_{A B} J_{A B}\right) \cdot\left(\iota_{L}\left(y^{-1}\right)^{T}\right)$, where $J_{A B}$ are generators of $\mathrm{SO}(8)$, is independent of $y$. Since

$$
\iota_{L}\left(y^{-1}\right)^{T}=\left(\begin{array}{cccccccc}
y_{0} & -y_{1} & -y_{2} & -y_{3} & -y_{4} & -y_{5} & -y_{6} & -y_{7} \\
y_{1} & y_{0} & -y_{3} & y_{2} & -y_{5} & y_{4} & -y_{7} & y_{6} \\
y_{2} & y_{3} & y_{0} & -y_{1} & -y_{6} & y_{7} & y_{4} & -y_{5} \\
y_{3} & -y_{2} & y_{1} & y_{0} & y_{7} & y_{6} & -y_{5} & -y_{4} \\
y_{4} & y_{5} & y_{6} & -y_{7} & y_{0} & -y_{1} & -y_{2} & y_{3} \\
y_{5} & -y_{4} & -y_{7} & -y_{6} & y_{1} & y_{0} & y_{3} & y_{2} \\
y_{6} & y_{7} & -y_{4} & y_{5} & y_{2} & -y_{3} & y_{0} & -y_{1} \\
y_{7} & -y_{6} & y_{5} & y_{4} & -y_{3} & -y_{2} & y_{1} & y_{0}
\end{array}\right)
$$

the vector $y \cdot\left(d_{A B} J_{A B}\right) \cdot \iota_{L}\left(y^{-1}\right)^{T}=\left(\begin{array}{llllllll}0 & c_{1} & c_{2} & c_{3} & c_{4} & c_{5} & c_{6} & c_{7}\end{array}\right)$ Altogether, independence of the $c_{i}$ with respect to $y$ leads to 21 independent constraints

$$
\begin{aligned}
d_{01} & =-d_{23}=-d_{45}=-d_{67} \\
d_{02} & =d_{13}=-d_{46}=d_{57} \\
d_{03} & =-d_{12}=d_{47}=d_{56} \\
d_{04} & =d_{15}=d_{26}=-d_{37} \\
d_{05} & =-d_{14}=-d_{27}=-d_{36} \\
d_{06} & =d_{17}=-d_{24}=d_{35} \\
d_{07} & =-d_{16}=d_{25}=d_{34}
\end{aligned}
$$

and $L_{g^{-1}{ }_{*}} g_{*} \cdot \xi$ is required to be in the 
seven-dimensional subspace spanned by the $\mathrm{SO}(8)$ generators $\left\{X_{i} \mid i=1, \ldots, 7\right\}$. This result can be traced to the fact that although $\iota_{R}(\tilde{g})$ and $\iota_{L}\left(y^{-1}\right)^{T}$ do not commute as elements of $\mathrm{SO}(8)$,

$$
y \cdot \iota_{R}(\tilde{g}) \cdot \iota_{L}\left(y^{-1}\right)^{T}=y \cdot \iota_{L}\left(y^{-1}\right)^{T} \cdot \iota_{R}(\tilde{g})
$$

because any two elements of the octonions form an associative algebra: $y^{-1}(y \cdot \tilde{g})=\left(y^{-1} y\right) \cdot \tilde{g}$ for $y, \tilde{g} \in \mathbb{O}$.

Let $v_{\mu}=\sigma_{*} \cdot \xi_{\mu} \in T_{\sigma(x)}(E)$. Then $\omega\left(v_{\mu}\right)=L_{y *}^{-1} \cdot \mathcal{V} v_{\mu}$ and

$$
\omega\left(R_{g *} v_{\mu}\right)=L_{(y \cdot g) *}^{-1} \mathcal{V}\left(R_{g *} v_{\mu}\right)
$$

If $v_{\mu}$ lies in the vertical subspace of $T_{\sigma(x)}(E), \mathcal{V}\left(R_{g *} v_{\mu}\right)=R_{g *} L_{y *} \omega\left(v_{\mu}\right)$. Representing left multiplication by a unit octonion $L_{y}$ as right multiplication by an $\mathrm{SO}(8)$ transformation, denoted by $R_{a_{y}}$, one finds that

$$
\omega\left(R_{g *} v_{\mu}\right)=R_{a_{(y \cdot g)}^{-1} *} R_{g *} R_{a_{y} *} \omega\left(v_{\mu}\right)=R_{\left(a_{y} g a_{y \cdot g}^{-1}\right) *} \omega\left(v_{\mu}\right)
$$

Defining $a_{y} g a_{y \cdot g}^{-1}$ to be $h(y, g) \in H$, the stability subgroup of $\mathrm{SO}(8)$ for the origin o in $S^{7}$, the connection form satisfies $\omega\left(R_{g *} v_{\mu}\right)=R_{h(y, g) *} \omega\left(v_{\mu}\right)$. Independence of the homogeneous part of the gauge transformation with respect to the fibre coordinate requires that $h(y, g)$ does not depend on $y$. If $g \leftrightarrow R_{g}^{T}=\left(c_{i j}\right), i, j=0,1, \ldots, 7, y$-independence of the homogeneous term is confirmed by a calculation of $\iota_{L}(y)^{T} R_{g}^{T}\left[\iota_{L}(y \cdot g)^{-1}\right]^{T}$. However,

$$
\begin{aligned}
\tilde{g}^{\prime} \iota_{L}(y)^{T}\left[\iota_{L}(y \cdot \tilde{g})^{-1}\right] & =\tilde{g}^{\prime T} \iota_{L}(y)^{T} \tilde{g} \iota_{L}\left(y^{-1}\right)^{T} \iota_{L}\left(\tilde{g}^{-1}\right)^{T} \\
& \leftrightarrow g^{-1} y^{-1}\left[\left(y \cdot \tilde{g}^{\prime}\right) \cdot \tilde{g}\right] \\
& \left.\neq g^{-1}\left(y^{-1}\left(y \cdot \tilde{g}^{\prime}\right) \cdot \tilde{g}\right)\right) \\
& =g^{-1}\left(\tilde{g}^{\prime} \cdot \tilde{g}\right)
\end{aligned}
$$

Thus, a $y$-dependent matrix is obtained when $R_{g}^{T}$ is given by $e^{t_{i} X_{i}}$ and a $y$-independent gauge transformation rule from the $\mathrm{SO}(8)$ action on an $S^{7}$ fibre therefore cannot be constructed.

The same procedure can also be applied to a bundle with $S^{7}$ fibre and an SU(4) structure group, allowing for the possibility of obtaining an action with the appropriate $\mathrm{SU}(3)$ gauge symmetry. The action of $\mathrm{SU}(4)$ on the seven-sphere follows from the invariance of the bilinear form $\bar{z}_{0}^{\prime} z_{0}+\bar{z}_{1}^{\prime} z_{1}+$ $\bar{z}_{2}^{\prime} z_{2}+\bar{z}_{3}^{\prime} z_{3}$, where $z=\left(\begin{array}{llll}z_{0} & z_{1} & z_{2} & z_{3}\end{array}\right) \in \mathbb{C}^{4}$. To define the equivalent of the inhomogeneous term in the transformation rule of the connection form, one needs the embedding of $S^{7}$ into SU(4). Left multiplication by a unit octonion maps any point $y^{\prime} \in S^{7}$ to a point $y^{\prime \prime}=y y^{\prime}$, and since a transitive group action on the sphere would take any pair of points into each other, left multiplication by the octonion $y$ can be represented as $\iota_{L}(y) \in S U(4)$, where $\iota_{L}: S^{7} \rightarrow S U(4)$.

The embedding of $\iota_{L}: S^{7} \rightarrow S U(4)$ will be defined so that

$$
\begin{aligned}
& \iota_{L}(y)\left(\begin{array}{l}
1 \\
0 \\
0 \\
0
\end{array}\right)=\left(\begin{array}{l}
y_{0}+i y_{1} \\
y_{2}+i y_{3} \\
y_{4}+i y_{5} \\
y_{6}+i y_{7}
\end{array}\right) \\
& \left(\begin{array}{llll}
1 & 0 & 0 & 0
\end{array}\right) \iota_{L}(y)^{T}=\left(y_{0}+i y_{1} y_{2}+i y_{3} y_{4}+i y_{5} y_{6}+i y_{7}\right)
\end{aligned}
$$


For simplicity, one may choose $\mathrm{g}$ to be $\iota_{L}(y)^{T}$ so that

$$
\begin{aligned}
& y_{0}+i y_{1}=g_{00}=\cos \theta_{2} \cos \theta_{1} \cos \phi_{1} e^{i \delta_{1}} \\
& y_{2}+i y_{3}=g_{01}=-\sin \theta_{2} e^{i\left(\delta_{1}-\sigma_{3}\right)} \\
& y_{4}+i y_{5}=g_{02}=-\cos \theta_{2} \sin \theta_{1} e^{i\left(\delta_{1}-\sigma_{2}\right)} \\
& y_{6}+i y_{7}=g_{03}=-\cos \theta_{2} \cos \theta_{1} \sin \phi_{1} e^{i\left(\delta_{1}-\sigma_{1}\right)}
\end{aligned}
$$

which clearly satisfies $\bar{y} y=1$. The proposition concerning the existence of SU(4) matrices mapping $o$ to $y$, or $y^{\prime}$ to $y^{\prime \prime}=y y^{\prime}$ is circumvented because the other entries of $g=\iota_{L}(y)^{T}$ are related nonlinearly to $g_{00}, g_{01}, g_{02}$ and $g_{03}$. Any SU(4) matrix with the first row given by (B8) will map $o$ to $y$. To simplify the calculations involving the entire matrix, the other eight parameters, $\delta_{2}, \delta_{3}, \phi_{2}, \phi_{3}, \theta_{3}, \sigma_{4}, \sigma_{5}, \sigma_{6}$ shall be set to zero. As $\iota_{L}\left(y^{-1}\right)^{T}=g^{\dagger}$,

$$
\begin{aligned}
& y \cdot\left(d_{A} J_{A}\right)\left(\iota_{L}\left(y^{-1}\right)^{T}\right) \equiv\left(\begin{array}{llll}
c_{0} & c_{1} & c_{2} & c_{3}
\end{array}\right) \\
& =\left(\cos \theta_{2} \cos \theta_{1} \cos \phi_{1} e^{i \delta_{1}}-\sin \theta_{2} e^{i\left(\delta_{1}-\sigma_{3}\right)}-\cos \theta_{2} \sin \theta_{1} e^{i\left(\delta_{1}-\sigma_{2}\right)}\right. \\
& \left.-\cos \theta_{2} \cos \theta_{1} \sin \phi_{1} e^{i\left(\delta_{1}-\sigma_{1}\right)}\right) \\
& \left(\begin{array}{cccc}
i d_{7} & d_{1}+i d_{4} & d_{2}+i d_{5} & d_{3}+i d_{6} \\
-d_{1}+i d_{4} & -i d_{7}+i d_{14} & d_{8}+i d_{9} & d_{10}+i d_{11} \\
-d_{2}+i d_{5} & -d_{8}+i d_{9} & -i d_{14}+i d_{15} & d_{12}+i d_{13} \\
-d_{3}+i d_{6} & -d_{10}+i d_{11} & -d_{12}+i d_{13} & -i d_{15}
\end{array}\right) \\
& \left(\begin{array}{ccc}
\cos \theta_{2} \cos \theta_{1} \cos \phi_{1} e^{-i \delta_{1}} & \sin \theta_{2} \cos \theta_{1} \cos \phi_{1} e^{-i \sigma_{3}} \\
-\sin \theta_{2} e^{-i\left(\delta_{1}-\sigma_{3}\right)} & \cos \theta_{2} \\
-\cos \theta_{2} \sin \theta_{1} e^{-i\left(\delta_{1}-\sigma_{2}\right)} & -\sin \theta_{2} \sin \theta_{1} e^{i\left(\sigma_{2}-\sigma_{3}\right)} \\
-\cos \theta_{2} \cos \theta_{1} \sin \phi_{1} e^{-i\left(\delta_{1}-\sigma_{1}\right)} & -\sin \theta_{2} \cos \theta_{1} \sin \phi_{1} e^{i\left(\sigma_{1}-\sigma_{3}\right)} \\
& \sin \theta_{1} \cos \phi_{1} e^{-i \sigma_{2}} & \sin \phi_{1} e^{i\left(\delta_{1}-\sigma_{1}\right)} \\
& 0 & 0 \\
\cos \theta_{1} & 0 \\
& -\sin \theta_{1} \sin \phi_{1} e^{i\left(\sigma_{1}-\sigma_{2}\right)} & \cos \phi_{1} e^{i \delta_{1}}
\end{array}\right)
\end{aligned}
$$

so that

$$
\begin{aligned}
c_{0} & =\left(\cos ^{2} \theta_{2} \cos ^{2} \theta_{1} \cos ^{2} \phi_{1}\right)\left(i d_{7}\right)+\sin ^{2} \theta_{2}\left(-i d_{7}+i d_{14}\right) \\
& +\left(\cos ^{2} \theta_{2} \sin ^{2} \theta_{1}\right)\left(-i d_{14}+i d_{15}\right)+\left(\cos ^{2} \theta_{2} \cos ^{2} \theta_{1} \sin ^{2} \phi_{1}\right)\left(-i d_{15}\right) \\
& +2 i\left(-\sin \theta_{2} \cos \theta_{2} \cos \theta_{1} \cos \phi_{1}\right)\left[d_{1} \sin \sigma_{3}+d_{4} \cos \sigma_{3}\right] \\
& +2 i\left(-\cos ^{2} \theta_{2} \sin \theta_{1} \cos \theta_{1} \cos \phi_{1}\right)\left[d_{2} \sin \sigma_{2}+d_{5} \cos \sigma_{2}\right] \\
& +2 i\left(-\cos ^{2} \theta_{2} \cos ^{2} \theta_{1} \sin \phi_{1} \sin \phi_{1}\right)\left[d_{3} \sin \sigma_{1}+d_{6} \cos \sigma_{1}\right] \\
& +2 i\left(\sin \theta_{2} \cos \theta_{2} \cos \theta_{1} \sin \phi_{1}\right)\left[d_{8} \sin \left(\sigma_{2}-\sigma_{3}\right)+d_{9} \cos \left(\sigma_{2}-\sigma_{3}\right)\right] \\
& +2 i\left(\sin \theta_{2} \cos \theta_{2} \cos \theta_{1} \sin \phi_{1}\right)\left[d_{10} \sin \left(\sigma_{1}-\sigma_{3}\right)+d_{11} \cos \left(\sigma_{1}-\sigma_{3}\right)\right] \\
& +2 i\left(\cos ^{2} \theta_{2} \sin \theta_{1} \cos \theta_{1} \sin \phi_{1}\right)\left[d_{12} \sin \left(\sigma_{1}-\sigma_{2}\right)+d_{13} \cos \left(\sigma_{1}-\sigma_{2}\right)\right]
\end{aligned}
$$

Independence of $c_{0}$ with respect to the angular coordinates requires

$$
\begin{aligned}
i d_{7} & =-i d_{7}+i d_{14}=-i d_{14}+i d_{15}=-i d_{15} \\
\sigma_{1} & =\sigma_{2}=\sigma_{3}=0 \\
d_{5} & =d_{6}=d_{9}=d_{11}=d_{13}=0
\end{aligned}
$$


The remaining coefficients are $d_{1}, d_{2}, d_{3}, d_{8}, d_{10}$ and $d_{12}$ and the fibre is restricted to a fourdimensional submanifold of $S^{7}, S U(2) \times U(1)$. Similarly,

$$
c_{1}=d_{1} \cos \theta_{1} \cos \phi_{1} e^{i \delta_{1}}+d_{8} \sin \theta_{1} e^{i \delta_{1}}+d_{10} \cos \theta_{1} \sin \phi_{1} e^{i \delta_{1}}
$$

which implies the vanishing of $d_{1}, d_{8}$ and $d_{10}$, and

$$
c_{2}=d_{2} \cos \theta_{2} \cos \phi_{1} e^{i \delta_{1}}+d_{12} \cos \theta_{2} \sin \phi_{1} e^{i \delta_{1}}
$$

which is independent of the angles if $d_{2}=d_{12}=0$. Finally,

$$
c_{3}=d_{3} \cos \theta_{2} \cos \theta_{1} e^{i \delta_{1}}
$$

so that independence with respect to the fibre coordinates can be achieved either by setting $d_{3}=0$ or $\theta_{1}=\theta_{2}=\delta_{1}=0$. The latter choice is obviously preferable as it still leaves a non-trivial action on the submanifold of the fibre parametrized by $\phi_{1}$, namely the action of U(1) on $S^{1}$.

Independence of the homogeneous part of the gauge transformation with respect to the fibre coordinates follows from the relation

$$
\left.L_{y}^{T} R_{g}^{T} L_{(y \cdot g)^{-1}}^{T}=\iota_{L}(y)^{T} \exp \left(d_{3} J_{3}\right)\left[\iota_{L}(y \cdot g)^{-1}\right)\right]^{T}=I d_{4}
$$

The remaining gauge symmetry is therefore associated with a $\mathrm{U}(1)$ gauge potential transforming as

$$
\begin{aligned}
& A_{\mu} \rightarrow A_{\mu}+\left(\partial_{\mu} g\right) g^{-1} \\
& A_{\mu}^{3} \rightarrow A_{\mu}^{3}+\partial_{\mu} d_{3}
\end{aligned}
$$

Constraints on the connection form transformation rule can also be obtained for bundles with fibres that are submanifolds of $S^{7}$. The structure group initially can be chosen to be any subgroup of $\mathrm{SO}(8)$ which preserves the submanifold. However, there also should be less conditions on the allowed transformations, because there are fewer fibre coordinates to be eliminated. Therefore, this leaves open the possibility of a residual gauge symmetry associated with the action of a subgroup of the original structure group on a submanifold of the standard fibre.

For the $S^{7}$ bundle, one choice for the submanifold is $S^{3}=\left\{y \in S^{7} \mid y_{0}^{2}+y_{1}^{2}+y_{2}^{2}+y_{3}^{2}=\right.$ $\left.1, y_{4}=y_{5}=y_{6}=y_{7}=0\right\}$. Independence of the inhomogeneous term, or equivalently $c_{1}, \ldots, c_{7}$, with respect to $y_{1}, \ldots, y_{3}$ leads to the 15 conditions

$$
\begin{aligned}
& d_{01}=-d_{23} \quad d_{02}=d_{13} \quad d_{03}=-d_{12} \\
& d_{04}=d_{15}=d_{26}=-d_{37} \\
& d_{05}=-d_{14}=-d_{27}=-d_{36} \\
& d_{06}=d_{17}=-d_{24}=d_{35} \\
& d_{07}=-d_{16}=d_{25}=d_{34}
\end{aligned}
$$

and the generators of the remaining 13 transformations are $J_{01}-J_{23}, J_{02}+J_{13}, J_{03}-J_{12}, J_{04}+J_{15}+$ $J_{26}-J_{37}, J_{05}-J_{14}-J_{27}-J_{36}, J_{06}+J_{17}-J_{24}+J_{35}, J_{07}-J_{16}+J_{25}+J_{34}, J_{45}, J_{46}, J_{47}, J_{56}, J_{57}$ and $J_{67}$. Independence of the homogeneous terms can be checked for these generators by establishing the $y$-independence of $L_{y}^{T} R_{g}^{T} L_{(y \cdot g)^{-1}}^{T}$, with $L_{y}^{T}$ given by (3.5) after replacing $y_{i}$ by $-y_{i}$ when $i=$ 
$1,2,3$ and setting $y_{j}=0, j \geq 4$, and $R_{g}^{T}$ equal to the exponential of the generator. This property can be verified for the first seven generators and holds trivially for the last six generators.

It is now necessary to note that the transformations generated by $J_{04}+J_{15}+J_{26}-J_{37}, J_{05}-$ $J_{14}-J_{25}-J_{36}, J_{06}+J_{17}-J_{24}+J_{35}$ and $J_{07}-J_{16}+J_{25}+J_{34}$ do not leave $S^{3}$ invariant and instead map it into four-dimensional submanifolds of $S^{7}$, consisting of a one-parameter family of three-spheres. The coordinates of these four-dimensional submanifolds are, respectively,

$\left(y_{0} \cos d_{04}, y_{1} \cos d_{04}, y_{2} \cos d_{04}, y_{3} \cos d_{04}, y_{0} \sin d_{04}, y_{1} \sin d_{04}\right.$

$$
\left.y_{2} \sin d_{04},-y_{3} \sin d_{04}\right)
$$

$\left(y_{0} \cos d_{05}, y_{1} \cos d_{05}, y_{2} \cos d_{05}, y_{3} \cos d_{05},-y_{1} \sin d_{05}, y_{0} \sin d_{05}\right.$,

$$
\left.-y_{3} \sin d_{05},-y_{2} \sin d_{05}\right)
$$

$\left(y_{0} \cos d_{06}, y_{1} \cos d_{06}, y_{2} \cos d_{06}, y_{3} \cos d_{06},-y_{2} \sin d_{06}, y_{3} \sin d_{06}\right.$,

$$
\left.y_{0} \sin d_{06}, y_{1} \sin d_{06}\right)
$$

$\left(y_{0} \cos d_{07}, y_{1} \cos d_{07}, y_{2} \cos d_{07}, y_{3} \cos d_{07}, y_{3} \sin d_{07}, y_{2} \sin d_{07}\right.$,

$$
\left.-y_{1} \sin d_{07}, y_{0} \sin d_{07}\right)
$$

and since there is a bijective, continuous map to the coordinates $\left\{\left(\left(y_{0}, y_{1}, y_{2}, y_{3}\right), \theta\right) \mid y_{0}^{2}+y_{1}^{2}+y_{2}^{2}+\right.$ $\left.y_{3}^{2}=1\right\}$, each of these submanifolds is topologically $S^{3} \times S^{1}$. Moreover, since the maps between the coordinates are also diffeomorphisms, one might consider constructing an $S^{3} \times S^{1}$ bundle.

However, the enlargement of the fibre from $S^{3}$ to $S^{3} \times S^{1}$ with non-zero entries in the last four components of the row vectors (3.22) implies that the quantities $c_{i}$ in (B1)-(B7) will not necessarily be independent of $y$. Three extra conditions

$$
\begin{aligned}
& d_{01}+d_{45}=d_{23}-d_{67} \\
& d_{02}+d_{46}=-d_{13}+d_{57} \\
& d_{03}-d_{47}=d_{12}+d_{56}
\end{aligned}
$$

must be satisfied before $c_{4}, \ldots, c_{7}$ are independent of the coordinates (3.22). There would then be 10 remaining generators $J_{01}-J_{23}+\alpha J_{45}-(\alpha+2) J_{67}, J_{02}+J_{13}+\beta J_{46}$ $+(\beta+2) J_{57}, J_{03}-J_{12}+\gamma J_{47}+(2-\gamma) J_{56}, J_{04}+J_{15}+J_{26}-J_{37}, J_{05}-J_{14}-J_{25}-J_{36}, J_{05}-$ $J_{14}-J_{25}-J_{36}, J_{06}+J_{17}-J_{24}+J_{35}, J_{07}-J_{16}+J_{25}+J_{34}, J_{45}-J_{67}, J_{46}+J_{57}$ and $J_{47}-J_{56}$.

The computation of $L_{y}^{T} R_{g}^{T} L_{(y \cdot g)^{-1}}^{T}$ must now be repeated for these 10 generators with the new coordinates (3.22) to determine whether the dependence on $y_{i}$ and $\theta$ can be eliminated in the homogeneous term in the transformation rule of the connection form. Denoting the coordinates of $S^{3} \times S^{1}$ by $y_{\theta}=y \cdot g_{0 l}(\theta), l=4,5,6,7$ so that

$$
\begin{aligned}
& g_{04}(\theta)=(\cos \theta, 0,0,0, \sin \theta, 0,0,0) \\
& g_{05}(\theta)=(\cos \theta, 0,0,0,0, \sin \theta, 0,0) \\
& g_{06}(\theta)=(\cos \theta, 0,0,0,0,0, \sin \theta, 0) \\
& g_{07}(\theta)=(\cos \theta, 0,0,0,0,0,0, \sin \theta)
\end{aligned}
$$

the following identity for alternative algebras [31]

$$
L_{y \cdot y^{\prime}}=L_{y} L_{y^{\prime}}+\left[L_{y}, R_{y^{\prime}}\right]
$$


implies that

$$
\begin{aligned}
L_{y \cdot g_{0 l}(\theta)}^{T} & =\left(L_{y} L_{g_{0 l}(\theta)}+L_{y} R_{g_{0 l}(\theta)}-R_{g_{0 l}(\theta)} L_{y}\right)^{T} \\
& =L_{g_{0 l}(\theta)}^{T} L_{y}^{T}+R_{g_{0 l}(\theta)}^{T} L_{y}^{T}-L_{y}^{T} R_{g_{0 l}(\theta)}^{T}
\end{aligned}
$$

Since

$$
\begin{aligned}
L_{\left(\left(y \cdot g_{0 l}(\theta)\right) \cdot R_{g}^{T}\right)^{-1} y^{\prime}} & =\left(\left(y \cdot g_{0 l}(\theta)\right) \cdot R_{g}^{T}\right)^{-1} y^{\prime}=\left(R_{g}^{T}\right)^{-1}\left(y \cdot g_{0 l}(\theta)\right)^{-1} y^{\prime} \\
& =\left(R_{g}^{T}\right)^{-1}\left(g_{0 l}(\theta)^{-1} \cdot y^{-1}\right) \cdot y^{\prime}=\left(R_{g}^{T}\right)^{-1} L_{g_{0 l}(\theta)^{-1} \cdot y^{-1}} \cdot y^{\prime}
\end{aligned}
$$

and

$$
L_{g_{0 l}(\theta)^{-1} \cdot y^{-1}}=L_{g_{0 l}(\theta)^{-1}} L_{y^{-1}}+L_{g_{0 l}(\theta)^{-1}} R_{y^{-1}}-R_{y^{-1}} L_{g_{0 l}(\theta)^{-1}}
$$

it can be shown that

$$
\begin{aligned}
& L_{y_{\theta}}^{T} R_{g}^{T} L_{\left(y_{\theta} \cdot g\right)^{-1}}^{T}=L_{y \cdot g_{0 l}(\theta)}^{T} R_{g}^{T} L_{\left(\left(y \cdot g_{0 l}(\theta)\right) \cdot g\right)^{-1}}^{T} \\
& =\left(L_{g_{0 l}(\theta)}^{T} L_{y}^{T}+R_{g_{0 l}(\theta)}^{T} L_{y}^{T}-L_{y}^{T} R_{g_{0 l}(\theta)}^{T}\right) R_{g}^{T} \\
& \left(L_{y^{-1}}^{T} L_{g_{0 l}(\theta)^{-1}}^{T}+R_{y^{-1}}^{T} L_{g_{0 l}(\theta)^{-1}}^{T}-L_{g_{0 l}(\theta)^{-1}}^{T} R_{y^{-1}}^{T}\right) R_{g}^{-1}
\end{aligned}
$$

The next step in determining whether the fibre-coordinate dependence can be eliminated from this expression would involve moving all of the $\theta$-dependent matrices to the center. Although it can be verified that $L_{g_{0 l}(\theta)} L_{y}=L_{y} L_{g_{0 l}(\theta)}$, the commutators $\left[L_{y}, R_{g_{0 l}(\theta)}\right]$ and $\left[R_{y}, L_{g_{0 l}(\theta)}\right]$ do not similarly vanish. Thus, the product (3.29) will be dependent on $y$ and $\theta$ for the general group element obtained by exponentiating the 10 generators listed above.

Consequently, to find any residual gauge symmetry, the coordinates must be restricted to the $S^{3}$ submanifold considered initially. From the above considerations, it follows that only 9 generators $J_{01}-J_{23}, J_{02}+J_{13}, J_{03}-J_{12}, J_{45}, J_{46}, J_{47}, J_{56}, J_{57}, J_{67}$ leave this submanifold invariant, and the last 6 generators act trivially on this three-sphere. As the remaining generators form an so(3) Lie algebra, there remains an $\mathrm{SO}(3)$ group of symmetry transformations acting on an $S^{3}$ fibre, which represents the gauge invariance of an $\mathrm{SO}(3)$ Yang-Mills theory corresponding to the $\mathrm{SO}(3)$ principal bundle.

When the submanifold is $S^{4}=\left\{y \in S^{7} \mid y_{0}^{2}+y_{1}^{2}+y_{2}^{2}+y_{3}^{2}+y_{4}^{2}=1, y_{5}=y_{6}=y_{7}=0\right\}$, independence of the inhomogenous term with respect to $y$ leads to 18 conditions

$$
\begin{aligned}
& d_{01}=-d_{23}=-d_{45} \\
& d_{02}=d_{13}=-d_{46} \\
& d_{03}=-d_{12}=d_{47} \\
& d_{04}=d_{15}=d_{26}=-d_{37} \\
& d_{05}=-d_{14}=-d_{27}=-d_{36} \\
& d_{06}=d_{17}=-d_{24}=d_{35} \\
& d_{07}=-d_{16}=d_{25}=d_{34}
\end{aligned}
$$

and the generators of the remaining ten generators are $J_{01}-J_{23}-J_{45}, J_{02}+J_{13}-J_{46}, J_{03}-J_{12}+J_{47}$, $J_{04}+J_{15}+J_{26}-J_{37}, J_{05}-J_{14}-J_{27}-J_{36}, J_{06}+J_{17}-J_{24}+J_{35}, J_{07}-J_{16}+J_{25}+J_{34}, J_{56}, J_{57}, J_{67}$. 
The exponentials of the generators $J_{01}-J_{23}-J_{45}, J_{02}+J_{13}-J_{46}$ and $J_{03}-J_{12}+J_{47}$ map $S^{4}$ into a five-dimensional submanifold diffeomorphic to $S^{4} \times S^{1}$, with coordinates

$$
\begin{gathered}
\left(y_{0} \cos d_{01}+y_{1} \sin d_{01},-y_{0} \sin d_{01}+y_{1} \cos d_{01}, y_{2} \cos d_{01}-y_{3} \sin d_{01},\right. \\
\left.y_{2} \sin d_{01}+y_{3} \cos d_{01}, y_{4} \cos d_{01}, y_{4} \sin d_{01}, 0,0\right) \\
\left(y_{0} \cos d_{02}+y_{2} \sin d_{02}, y_{1} \cos d_{02}+y_{3} \sin d_{02},-y_{0} \sin d_{02}+y_{3} \cos d_{02},\right. \\
\left.-y_{1} \sin d_{02}+y_{3} \cos d_{02}, y_{4} \cos d_{02}, 0, y_{4} \sin d_{02}, 0\right) \\
\left(y_{0} \cos d_{03}+y_{3} \sin d_{03}, y_{1} \cos d_{03}-y_{2} \sin d_{03}, y_{1} \sin d_{03}+y_{3} \cos d_{03},\right. \\
\left.-y_{0} \sin d_{03}+y_{3} \cos d_{03}, y_{4} \cos d_{03}, 0,0,-y_{4} \sin d_{03}\right)
\end{gathered}
$$

The transformations obtained by exponentiating the four generators $J_{04}+J_{15}+J_{26}-J_{37}, J_{05}-$ $J_{14}-J_{27}-J_{36}, J_{06}+J_{17}-J_{24}+J_{35}, J_{07}-J_{16}+J_{25}+J_{34}$ map $S^{4}$ into a five-dimensional submanifold diffeomorphic to $S^{4} \times S^{1}$, with coordinates

$$
\begin{gathered}
\left(y_{0} \cos d_{04}-y_{4} \sin d_{04}, y_{1} \cos d_{04}, y_{2} \cos d_{04}, y_{3} \cos d_{04},\right. \\
\left.y_{0} \sin d_{04}+y_{4} \cos d_{04}, y_{1} \sin d_{04}, y_{2} \sin d_{04},-y_{3} \sin d_{04}\right) \\
\left(y_{0} \cos d_{05}, y_{1} \cos d_{05}-y_{4} \sin d_{05}, y_{2} \cos d_{05}, y_{3} \cos d_{05},\right. \\
\left.y_{1} \sin d_{05}+y_{4} \cos d_{05}, y_{0} \sin d_{05},-y_{3} \sin d_{05},-y_{2} \sin d_{05}\right) \\
\left(y_{0} \cos d_{06}, y_{1} \cos d_{06}, y_{2} \cos d_{06}-y_{4} \sin d_{06}, y_{3} \cos d_{06},\right. \\
\left.y_{2} \sin d_{06}+y_{4} \cos d_{06},-y_{3} \sin d_{06},-y_{0} \sin d_{06},-y_{1} \sin d_{06}\right) \\
\left(y_{0} \cos d_{07}, y_{1} \cos d_{07}, y_{2} \cos d_{07}, y_{3} \cos d_{07}+y_{4} \sin d_{07},\right. \\
\left.-y_{3} \sin d_{07}+y_{4} \cos d_{07},-y_{2} \sin d_{07}, y_{1} \sin d_{07},-y_{0} \sin d_{07}\right)
\end{gathered}
$$

It can be confirmed that $L_{y}^{T} R_{g}^{T} L_{(y \cdot g)^{-1}}^{T}$ depends on $y$ for each set of coordinates. Consequently, there is no immediate residual symmetry group in the sense of equation (3.2) when the submanifold is chosen to be $S^{4}$.

A U(1) symmetry can be deduced by embedding $S^{4}$ in $\mathrm{SU}(3)$, and restricting the coordinatization of the $\mathrm{SU}(4)$ transformation in $\S 3$ to this subgroup. The derivation of this symmetry group for $S^{4}$ and $S^{5}$ are similar. The condition of independence of the connection form with respect to the fibre coordinate implies that the dimension of the symmetry group associated with the transformation rule (3.2) must remain constant or decrease as the dimension of the submanifold defining the fibre increases.

When the submanifold is $S^{5}$, independence of the inhomogeneous term with respect to the fibre coordinate gives rise to 20 conditions leaving 8 generators. The action of these generators on $\left(y_{0}, y_{1}, y_{2}, y_{3}, y_{4}, y_{5}, 0,0,0\right)$ produces a new set of coordinates and the homogeneous term depends on $y$ for each of these coordinates. This follows from the results for $S^{4}$, as the restriction of the homogeneous term to $y_{5}=0$ already reveals a dependence on $y$.

The embedding of $S^{5}$ into $S U(3)$ can be deduced from the earlier embedding of $S^{7}$ into $S U(4)$ by setting $\delta_{1}+\delta_{2}+\delta_{3}=\phi_{3}=\sigma_{6}=\phi_{2}=\sigma_{1}=\phi_{1}=\sigma_{1}=0$. The remaining parameters are $\theta_{1}, \theta_{2}, \theta_{3}, \sigma_{2}, \sigma_{3}, \sigma_{5}, \delta_{1}, \delta_{2}$ and the entries of the general $\mathrm{SU}(3)$ matrix in Euler angles, 


$$
\begin{aligned}
D\left(\delta_{1}, \delta_{2}-\delta_{1}\right. & \left.-\delta_{2}\right) U_{23}\left(\theta_{3}, \sigma_{5}\right) U_{12}\left(\theta_{2}, \sigma_{3}\right) U_{13}\left(\theta_{1}, \sigma_{2}\right) \text { is } \\
g_{00} & =\cos \theta_{2} \cos \theta_{1} e^{i \delta_{1}} \\
g_{01} & =-\sin \theta_{2} e^{i\left(\delta_{1}-\delta_{3}\right)} \\
g_{02} & =-\cos \theta_{2} \sin \theta_{1} e^{i\left(\delta_{1}-\sigma_{2}\right)} \\
g_{10} & =\cos \theta_{3} \cos \theta_{2} \sin \theta_{2} \cos \theta_{1} e^{i\left(\delta_{2}+\sigma_{3}\right)}-\sin \theta_{3} \sin \theta_{1} e^{i\left(\sigma_{2}+\delta_{2}-\sigma_{3}\right)} \\
g_{11} & =\cos \theta_{2} \cos \theta_{3} e^{i \delta_{2}} \\
g_{12} & =-\cos \theta_{3} \sin \theta_{2} \sin \theta_{1} e^{i\left(\delta_{2}+\sigma_{3}-\sigma_{2}\right)}-\sin \theta_{3} \cos \theta_{1} e^{i\left(\delta_{2}-\sigma_{5}\right)} \\
g_{20} & =\sin \theta_{3} \sin \theta_{2} \cos \theta_{1} e^{i\left(-\delta_{1}-\delta_{2}+\sigma_{3}+\sigma_{5}\right)}+\cos \theta_{3} \sin \theta_{1} e^{i\left(\sigma_{2}-\delta_{1}-\delta_{2}\right)} \\
g_{21} & =\sin \theta_{3} \cos \theta_{2} e^{i\left(\sigma_{5}-\delta_{1}-\delta_{2}\right)} \\
g_{22} & =-\sin \theta_{3} \sin \theta_{2} \sin \theta_{1} e^{i\left(-\delta_{1}-\delta_{2}+\sigma_{3}+\sigma_{5}-\sigma_{2}\right)}
\end{aligned}
$$

Restriction to $S^{5}$ is obtained by setting $\theta_{3}=\sigma_{5}=\delta_{2}=0$ and using the map $\iota_{L}: S^{5} \rightarrow S U(3)$

$$
\begin{aligned}
\iota_{L}(y) & =\left(\begin{array}{l}
1 \\
0 \\
0
\end{array}\right)=\left(\begin{array}{l}
y_{0}+i y_{1} \\
y_{2}+i y_{3} \\
y_{4}+i y_{5}
\end{array}\right) \\
& =\left(\begin{array}{c}
\cos \theta_{2} \cos \theta_{1} e^{i \delta_{1}} \\
-\sin \theta_{2} e^{i\left(\delta_{1}-\sigma_{3}\right)} \\
-\cos \theta_{2} \sin \theta_{1} e^{i\left(\delta_{1}-\sigma_{2}\right)}
\end{array}\right)
\end{aligned}
$$

If $\left(c_{0}, c_{1}, c_{2}\right) \equiv y \cdot\left(d_{A} J_{A}\right)\left(\iota_{L}\left(y^{-1}\right)^{T}\right)$, independence of $c_{0}$ with respect to the fibre coordinates implies that $d_{5}=d_{7}=d_{9}=d_{14}=0$ and $\sigma_{2}=\sigma_{3}=0$. The remaining coefficients are $d_{1}, d_{2}, d_{8}$. Since $c_{1}=d_{1} \cos \theta_{1} e^{i \delta_{1}}+d_{8} \sin \theta_{1} e^{i \delta_{1}}$, independence with respect to the fibre coordinate $y$ implies that $d_{1}=d_{8}=0$ and $c_{2}=d_{2} \cos \theta_{2} e^{i \delta_{1}}$. If $\theta_{2}=\delta_{1}=0$, there is still a non-trivial U(1) action parametrized by $\theta_{1}$. This is consistent with the existence of a $\mathrm{U}(1)$ affinor structure on $S^{5}$ [37].

Similarly, independence of the inhomogenous term for an $S^{6}$ submanifold implies 21 conditions and leaves 7 remaining generators. Dependence of the homogeneous term for the coordinates generated by the action of these generators on $\left(y_{0}, y_{1}, y_{2}, y_{3}, y_{4}, y_{5}, y_{6}, 0\right)$ again can be verified.

When $\mathrm{G} / \mathrm{H}$ is a reductive homogeneous space, the G-invariant affine connections are in oneto-one correspondence with the set of algebra multiplications on $(\mathbf{m}, *)$ with $(\mathbf{g}, \mathbf{h})$ a reductive pair, $\mathbf{g}=\mathbf{m} \oplus \mathbf{h}$ and $A d H \subseteq A u t(\mathbf{m}, *)$ where $\mathbf{g}, \mathbf{h}$ are Lie algebras of $\mathrm{G}$ and $\mathrm{H}$, where $G / H$ is a reductive homogeneous space [38], and this property has been used to characterize $G_{2}$-invariant affine connections on $S^{6}=G_{2} / S U(3)$ [39] and Spin(7)-invariant connections on $S^{7}=\operatorname{Spin}(7) / G_{2}$ [40]. The affine connection may take values in the vector space representing the vertical tangent space, but the torsion and curvature forms are acting on vectors in this vector space. A connection form may be introduced for the total bundle by extending evaluation of the form to the horizontal lifts of vectors in the base space and the projection of tangent vectors to the sections of the total space onto the the vertical subspace. While the transformation of these connection forms would be of the standard type, the connections defined in this manner would generally depend on the fibre coordinates. Invariants for the groups $G_{2}$ and $\operatorname{Spin}(7)$ also have been defined [41][42], but they consist of trace polynomials rather than the gauge potentials and the transformation rule does not contain the inhomogeneous terms required for connections. 
Beginning with an $\mathrm{SO}(8)$ structure group, it appeared possible that an allowed symmetry group larger than the fibre could be obtained if the fibre was chosen to be an appropriate submanifold of $S^{7}$. The computation of the fibre coordinate dependence of the connection form transformation rule for the $S^{3}$ submanifold demonstrates that the constraints reduce the structure group to $\mathrm{SO}(3)$, producing the standard principal bundle structure. This property is verified for general bundles in Appendix A.

\section{Gauge Potentials and Projections of the Tangent Vector Fields to the $S^{7}$ Fibre onto Submanifolds}

Given a diffeomorphism between an $\mathrm{SU}(2)$ bundle over $M_{4} \times S^{4}$ and an $S^{7}$ bundle over $M_{4}$, it follows that a change of section in the $S^{7}$ bundle would mix fibre coordinates in the $\mathrm{SU}(2)$ bundle over eight-dimensional base space. For a fibre admitting a global parallelism, projection of the connection transformation rule to the base manifold could give rise to a generalized transformation law in eight dimensions. The action of octonion multiplication can be projected onto a parallelism on $\mathrm{SU}(2)$, and it may be anticipated that this parallelism on the $\mathrm{SU}(2)$ fibre can be used to express the terms in the transformed connection, $T \psi_{\beta \alpha}^{y}\left(\xi_{x}\right)$, as $\phi_{y^{\prime}} W(x)$, and $T \psi_{\beta \alpha}^{x} \cdot \Gamma^{\alpha}\left(\xi_{x}, y\right)$, as $\phi_{y^{\prime}} \cdot A(x) \cdot \Gamma^{\alpha}\left(\xi_{x}\right)$. The local diffeomorphism between $S^{7}$ and $S^{4} \times S^{3}$, $\psi_{U}:\left.\left.S^{7}\right|_{U} \rightarrow\left(S^{4} \times S^{3}\right)\right|_{\psi(U)}$ induces a map between the vector fields $\psi_{*}:\left.\left.T S^{7}\right|_{U} \rightarrow T\left(S^{4} \times S^{3}\right)\right|_{\psi(U)}$ defined by $\psi_{*}\left(e_{1}(y), \ldots, e_{7}(y)\right)=\left(\left(e_{1}^{\prime}(\psi(y)), \ldots, e_{4}^{\prime}(\psi(y)), \ldots,\left(e_{1}^{\prime \prime}(\psi(y)), \ldots, e_{3}^{\prime \prime}(\psi(y))\right)\right.\right.$. While there are seven independent smooth vector fields on the fibre, the image of the tangent mapping is a set of vector fields, which can be projected locally to linear combinations of three vector fields on an $S^{3}$ submanifold.

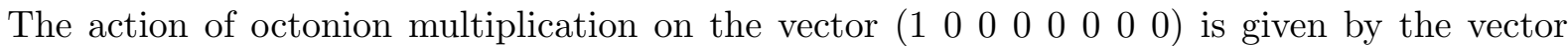

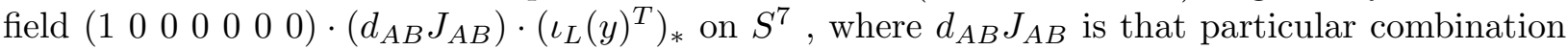
of generators in the Lie algebra of $\mathrm{SO}(8)$ belonging to the seven-dimensional subspace spanned by $\left\{X_{i}, i=1, \ldots, 7\right\}$. The product $y \cdot\left(d_{A B} J_{A B}\right) \cdot\left(\iota_{L}\left(y^{-1}\right)^{T}\right)_{*}$ has been shown to be independent of $y$ in $\S 3$, and the same property holds for $y^{-1} \cdot\left(d_{A B} J_{A B}\right) \cdot\left(\iota_{L}(y)^{T}\right)_{*}$. For a given value of $y$, only three independent non-zero vectors $\left(\begin{array}{lllllllll}0 & 0 & 0 & 0 & y_{5} & -y_{4} & y_{7} & - & -y_{6}\end{array}\right),\left(\begin{array}{llllllll}0 & 0 & 0 & 0 & y_{6} & -y_{7} & -y_{4} & y_{5}\end{array}\right)$ and $\left(\begin{array}{lllllll}0 & 0 & 0 & 0 & -y_{7}-y_{6} & y_{5} & y_{4}\end{array}\right)$ are obtained when $y_{0}, y_{1}, y_{2}, y_{3}$ are set equal to zero. However, it has been established that the four generators in $\mathrm{SO}(8)$ representing $X_{i}, i=4, \ldots, 7$ do not leave this $S^{3}$ submanifold invariant, and the projection of these four vectors onto $S^{3}$ can only be computed when the coordinates $y_{i}, i=0,1,2,3$ are allowed to be non-zero. The group elements

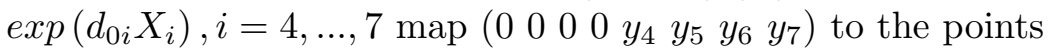

$\left(y_{4} \sin d_{04}, y_{5} \sin d_{04}, y_{6} \sin d_{04},-y_{7} \sin d_{04}, y_{4} \cos d_{04}, y_{5} \cos d_{04}\right.$,

$\left.y_{6} \cos d_{04}, y_{7} \cos d_{04}\right)$,

$\left(y_{5} \sin d_{05},-y_{4} \sin d_{05},-y_{7} \sin d_{05},-y_{6} \sin d_{05}, y_{4} \cos d_{05}, y_{5} \cos d_{05}\right.$,

$\left.y_{6} \cos d_{05}, y_{7} \cos d_{05}\right)$

$\left(y_{6} \sin d_{06}, y_{7} \sin d_{06},-y_{4} \sin d_{06}, y_{5} \sin d_{06}, y_{4} \cos d_{06}, y_{5} \cos d_{06}\right.$,

$\left.y_{6} \cos d_{06}, y_{7} \cos d_{06}\right)$

$\left(y_{7} \sin d_{07},-y_{6} \sin d_{07}, y_{5} \sin d_{07}, y_{4} \sin d_{07}, y_{4} \cos d_{07}, y_{5} \cos d_{07}\right.$,

$\left.y_{6} \cos d_{07}, y_{7} \cos d_{07}\right)$ 
The seven tangent vectors $\left(\begin{array}{lllllll}1 & 0 & 0 & 0 & 0 & 0 & 0\end{array}\right) \cdot X_{i} \cdot\left(\iota_{L}\left(y^{\prime}\right)^{T}\right)_{*}$ are given by

$$
\begin{aligned}
& \left(-y_{1}^{\prime} y_{0}^{\prime} y_{3}^{\prime}-y_{2}^{\prime} y_{5}^{\prime}-y_{4}^{\prime} y_{7}^{\prime}-y_{6}^{\prime}\right) \\
& \left(-y_{2}^{\prime}-y_{3}^{\prime} y_{0}^{\prime} y_{1}^{\prime} y_{6}^{\prime}-y_{7}^{\prime}-y_{4}^{\prime} y_{5}^{\prime}\right) \\
& \left(-y_{3}^{\prime} y_{2}^{\prime}-y_{1}^{\prime} y_{0}^{\prime}-y_{7}^{\prime}-y_{6}^{\prime} y_{5}^{\prime} y_{4}^{\prime}\right) \\
& \left(-y_{4}^{\prime}-y_{5}^{\prime}-y_{6}^{\prime} y_{7}^{\prime} y_{0}^{\prime} y_{1}^{\prime} y_{2}^{\prime}-y_{3}^{\prime}\right) \\
& \left(-y_{5}^{\prime} y_{4}^{\prime} y_{7}^{\prime} y_{6}^{\prime}-y_{1}^{\prime} y_{0}^{\prime}-y_{3}^{\prime}-y_{2}^{\prime}\right) \\
& \left(-y_{6}^{\prime}-y_{7}^{\prime} y_{4}^{\prime}-y_{5}^{\prime}-y_{2}^{\prime} y_{3}^{\prime} y_{0}^{\prime} y_{1}^{\prime}\right) \\
& \left(-y_{7}^{\prime} y_{6}^{\prime}-y_{5}^{\prime}-y_{4}^{\prime} y_{3}^{\prime} y_{2}^{\prime}-y_{1}^{\prime} y_{0}^{\prime}\right)
\end{aligned}
$$

and they can be evaluated at each of the four points (4.1). Consider, in particular, the four vectors

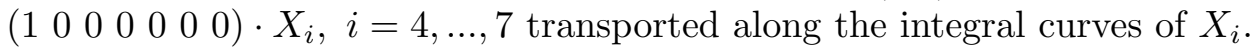

$\left(-y_{4} \cos d_{04},-y_{5} \cos d_{04},-y_{6} \cos d_{04}, y_{7} \cos d_{04}, y_{4} \sin d_{04}, y_{5} \sin d_{04}\right.$, $\left.y_{6} \sin d_{04}, y_{7} \sin d_{04}\right)$

$\left(-y_{5} \cos d_{05}, y_{4} \cos d_{05}, y_{7} \cos d_{05}, y_{6} \cos d_{05}, y_{4} \sin d_{05}, y_{5} \sin d_{05}\right.$, $\left.y_{6} \sin d_{05}, y_{7} \sin d_{05}\right)$

$\left(-y_{6} \cos d_{06},-y_{7} \cos d_{06}, y_{4} \cos d_{06},-y_{5} \cos d_{06}, y_{4} \sin d_{06}, y_{5} \sin d_{06}\right.$, $\left.y_{6} \sin d_{06}, y_{7} \sin d_{06}\right)$

$\left(-y_{7} \cos d_{07}, y_{6} \cos d_{07},-y_{5} \cos d_{07},-y_{4} \cos d_{07}, y_{4} \sin d_{07}, y_{5} \sin d_{07}\right.$, $\left.y_{6} \sin d_{07}, y_{7} \sin d_{07}\right)$

The projections onto the last four components of these eight-component vectors are identical and represent the normal vector to the three sphere spanned by coordinates $\left(0,0,0,0, y_{4}, y_{5}, y_{6}, y_{7}\right)$, thus vanishing in the tangent plane. Given a normal vector $\vec{n}$ to the tangent plane in the eightdimensional embedding space which defines a perpendicular from the vector $\vec{v}$ to the tangent plane, and a decomposition $k_{1} \vec{t}+k_{2} \vec{n}=\vec{v}$, the projection onto this tangent vector would be $\frac{(\vec{v} \cdot \vec{t}) \vec{t}}{|\vec{t}|^{2}}$. The tangent plane to the three sphere at the point $\left(y_{4} \sin d_{04}, y_{5} \sin d_{04}, y_{6} \sin d_{04},-y_{7} \sin d_{04}, y_{4} \cos d_{04}\right.$ $\left.y_{5} \cos d_{04}, y_{6} \cos d_{04}, y_{7} \cos d_{04}\right)$ is spanned by the three vectors

$$
\begin{aligned}
& \vec{t}_{4}^{(4)}=\left(y_{7} \sin d_{04}, 0,0, y_{4} \sin d_{04}, y_{7} \cos d_{04}, 0,0,-y_{4} \cos d_{04}\right) \\
& \vec{t}_{5}^{(4)}=\left(0, y_{7} \sin d_{04}, 0, y_{5} \sin d_{04}, 0, y_{7} \cos d_{04}, 0,-y_{5} \cos d_{04}\right) \\
& \vec{t}_{6}^{(4)}=\left(0,0, y_{7} \sin d_{04}, y_{6} \sin d_{04}, 0,0, y_{7} \cos d_{04},-y_{6} \cos d_{04}\right)
\end{aligned}
$$

The normal vectors to the submanifolds (4.3) within the tangent space to the seven-sphere can be deduced from four orthogonality conditions. In the first instance, these normal vectors are

$$
\begin{aligned}
& \vec{n}_{0}^{(4)}=\left(\cos d_{04}, 0,0,0,-\sin d_{04}, 0,0,0\right) \\
& \vec{n}_{1}^{(4)}=\left(0, \cos d_{04}, 0,0,0,-\sin d_{04}, 0,0\right) \\
& \vec{n}_{2}^{(4)}=\left(0,0, \cos d_{04}, 0,0,0,-\sin d_{04}, 0\right) \\
& \vec{n}_{3}^{(4)}=\left(0,0,0, \cos d_{04}, 0,0,0, \sin d_{04}\right)
\end{aligned}
$$


The equivalence of $c_{0} \vec{n}_{0}^{(4)}+c_{1} \vec{n}_{1}^{(4)}+c_{2} \vec{n}_{2}^{(4)}+c_{3} \vec{n}_{3}^{(4)}+\lambda_{4} \vec{t}_{4}^{(4)}+\lambda_{5} \vec{t}_{5}^{(4)}+\lambda_{6} \vec{t}_{6}^{(4)}$ with the initial vector in the set (4.2) requires $c_{0}=-y_{4}, c_{1}=-y_{5}, c_{2}=-y_{6}, c_{3}=y_{7}$. The projection along the normal vector $-y_{4} \vec{n}_{0}^{(4)}-y_{5} \vec{n}_{1}^{(4)}-y_{6} \vec{n}_{2}^{(4)}+y_{7} \vec{n}_{3}^{(4)}$ vanishes.

Based on the coordinates for the second submanifold, the tangent vectors are

$$
\begin{aligned}
& \vec{t}_{4}^{(5)}=\left(0,-y_{7} \sin d_{05}, y_{4} \sin d_{05}, 0, y_{7} \cos d_{05}, 0,0,-y_{4} \cos d_{05}\right) \\
& \vec{t}_{5}^{(5)}=\left(y_{7} \sin d_{05}, 0, y_{5} \sin d_{05}, 0,0, y_{7} \cos d_{05}, 0,-y_{5} \cos d_{05}\right) \\
& \vec{t}_{6}^{(5]}=\left(0,0, y_{6} \sin d_{05},-y_{7} \sin d_{05}, 0,0, y_{7} \cos d_{05},-y_{6} \cos d_{05}\right)
\end{aligned}
$$

From the orthogonality relations, the normal vectors

$$
\begin{aligned}
& \left(0, \cos d_{05}, 0,0, \sin d_{05}, 0,0,0\right) \\
& \left(\cos d_{05}, 0,0,0,0,-\sin d_{05}, 0,0\right) \\
& \left(0,0,0, \cos d_{05}, 0,0, \sin d_{05}, 0\right) \\
& \left(0, \cos d_{05}, 0,0,0,0,0, \sin d_{05}\right)
\end{aligned}
$$

The second vector in the set (4.2) can be obtained from the linear combination $c_{0}^{(5)} \vec{n}_{0}^{(5)}+c_{1}^{(5)} \vec{n}_{1}^{(5)}+$ $c_{2}^{(5)} \vec{n}_{2}^{(5)}+c_{3}^{(5)} \vec{n}_{3}^{(5)}+\lambda_{4}^{(5)} \vec{t}_{4}^{(5)}+\lambda_{5}^{(5)} \vec{t}_{5}^{(5)}+\lambda_{6}^{(5)} \vec{t}_{6}^{(5)}$,

$$
\begin{aligned}
c_{1}^{(5)} \cos d_{05}+\lambda_{5}^{(5)} y_{7} \sin d_{05} & =-y_{5} \cos d_{05} \\
c_{0}^{(5)} \cos d_{05}-\lambda_{4}^{(5)} y_{7} \sin d_{05} & =y_{4} \cos d_{05} \\
c_{2}^{(5)} \cos d_{05}+\left(\lambda_{4}^{(5)} y_{4}+\lambda_{5}^{(5)} y_{5}+\lambda_{6}^{(5)} y_{6}\right) \sin d_{05} & =y_{7} \cos d_{05} \\
c_{3}^{(5)} \cos d_{05}-\lambda_{6}^{(5)} y_{7} \sin d_{05} & =y_{6} \cos d_{05} \\
c_{0}^{(5)} \sin d_{05}+\lambda_{4}^{(5)} y_{7} \cos d_{05} & =y_{4} \sin d_{05} \\
-c_{1}^{(5)} \sin d_{05}+\lambda_{5}^{(5)} y_{7} \cos d_{05} & =y_{5} \sin d_{05} \\
c_{2}^{(5)} \sin d_{05}+\lambda_{6}^{(5)} \cos d_{05} & =y_{6} \cos d_{05} \\
c_{3}^{(5)} \sin d_{05}-\left(\lambda_{4}^{(5)} y_{4}+\lambda_{5}^{(5)} y_{5}+\lambda_{6}^{(5)} y_{6}\right) \cos d_{05} & =y_{7} \sin d_{05}
\end{aligned}
$$

which has the solution $c_{0}^{(5)}=y_{4}, c_{1}^{(5)}=-y_{5}, c_{2}^{(5)}=y_{6}, c_{3}^{(5)}=y_{7}, \lambda_{4}^{(5)}=\lambda_{5}^{(5)}=\lambda_{6}^{(5)}=0$.

At the point $\left(y_{6} \sin d_{06}, y_{7} \sin d_{06},-y_{4} \sin d_{06}, y_{5} \sin d_{06}, y_{4} \cos d_{06}, y_{5} \cos d_{06}\right.$, $\left.y_{6} \cos d_{06}, y_{7} \cos d_{06}\right)$, the tangent vectors are

$$
\begin{aligned}
& \vec{t}_{4}^{(6)}=\left(0,-y_{4} \sin d_{06},-y_{7} \sin d_{06}, 0, y_{7} \cos d_{06}, 0,-y_{4} \cos d_{06}\right) \\
& \vec{t}_{5}^{(6)}=\left(0,-y_{5} \sin d_{06}, 0, y_{7} \sin d_{06}, 0, y_{7} \cos d_{06}, 0,-y_{5} \cos d_{06}\right) \\
& \vec{t}_{6}^{(6)}=\left(y_{7} \sin d_{06},-y_{6} \sin d_{06}, 0,0,0,0, y_{7} \cos d_{06},-y_{6} \cos d_{06}\right)
\end{aligned}
$$

The normal vectors are

$$
\begin{aligned}
& \left(0,0, \cos d_{06}, 0, \sin d_{06}, 0,0,0\right) \\
& \left(0,0,0, \cos d_{06}, 0,-\sin d_{06}, 0,0\right) \\
& \left(\cos d_{06}, 0,0,0,0,0,-\sin d_{06}, 0\right) \\
& \left(0, \cos d_{06}, 0,0,0,0,0-\sin d_{06}\right)
\end{aligned}
$$


The third vector in the set (4.2) can be obtained from a linear combination of the four normal vectors and three tangent vectors by imposing the conditions by setting $c_{0}^{(6)}=y_{4}, c_{1}^{(6)}=-y_{5}, c_{2}^{(6)}=$ $-y_{6}, c_{3}^{(6)}=-y_{7}, \lambda_{4}^{(6)}=\lambda_{5}^{(6)}=\lambda_{6}^{(6)}=0$.

The tangent vectors at the point $\left(y_{7} \sin d_{07},-y_{6} \sin d_{07},-y_{5} \sin d_{07}, y_{4} \sin d_{07}\right.$, $\left.y_{4} \cos d_{07} y_{5} \cos d_{07}, y_{6} \cos d_{07}, y_{7} \cos d_{07}\right)$, are

$$
\begin{aligned}
& t_{4}^{(7)}=\left(-y_{4} \sin d_{07}, 0,0,0, y_{7} \sin d_{07}, y_{7} \cos d_{07}, 0,0,-y_{4} \cos d_{07}\right) \\
& t_{5}^{(7)}=\left(-y_{5} \sin d_{07}, 0, y_{7} \sin d_{07}, 0,0, y_{7} \cos d_{07}, 0,-y_{5} \cos d_{07}\right) \\
& t_{6}^{(7)}=\left(-y_{6} \sin d_{07},-y_{7} \sin d_{07}, 0,0,0,0, y_{7} \cos d_{07},-y_{6} \cos d_{07}\right)
\end{aligned}
$$

and the normal vectors are

$$
\begin{aligned}
& \left(0,0,0, \cos d_{07},-\sin d_{07}, 0,0,0\right) \\
& \left(0,0, \cos d_{07}, 0,0,-\sin d_{07}, 0,0\right) \\
& \left(0, \cos d_{07}, 0,0,0,0, \sin d_{07}, 0\right) \\
& \left(\cos d_{07}, 0,0,0,0,0,0,-\sin d_{07}\right)
\end{aligned}
$$

and equality between $c_{0}^{(7)} \vec{n}_{0}^{(7)}+c_{1}^{(7)} \vec{n}_{1}^{(7)}+c_{2}^{(7)} \vec{n}_{2}^{(7)}+c_{3}^{(7)} \vec{n}_{3}^{(7)}+\lambda_{4}^{(7)} \vec{t}_{4}^{(7)}+\lambda_{5}^{(7)} \vec{t}_{5}^{(7)}+\lambda_{6}^{(7)} \vec{t}_{6}^{(7)}$ and the vector $\left(-y_{7} \cos d_{07}, y_{6} \cos d_{07},-y_{5} \cos d_{07},-y_{4} \cos d_{07}, y_{4} \sin d_{07}, y_{5} \cos d_{07}, y_{6} \cos d_{07}\right.$, $\left.y_{7} \cos d_{07}\right)$ is obtained if $c_{0}^{(7)}=-y_{4}, c_{1}^{(7)}=-y_{5}, c_{2}^{(7)}=y_{6}, c_{3}^{(7)}=-y_{7}, \lambda_{4}^{(7)}=\lambda_{5}^{(7)}=\lambda_{6}^{(7)}=0$.

Since the orthogonality of the vectors $X_{i}$ will be preserved by the $S^{7}$ parallelism, the transport of other vectors $X_{k}, k \neq i, k=4, \ldots, 7$ again will have the property of vanishing projection along the normal vectors to the $S^{3}$ submanifold.

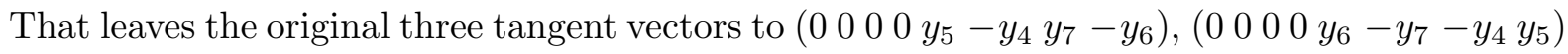
and $\left(\begin{array}{lllllll}0 & 0 & 0 & 0 & -y_{7}-y_{6} & y_{5} & y_{4}\end{array}\right)$ so that an $S O(3)$ gauge theory initially would be obtained upon projection to the $S^{3}$ submanifold. form

However, upon setting $y_{0}, y_{1}, y_{2}, y_{3}$ equal to zero, the seven tangent vectors at $y$ take the $\left(0,0,0,0, y_{5},-y_{4}, y_{7},-y_{6}\right),\left(0,0,0,0, y_{6},-y_{7},-y_{4}, y_{5}\right),\left(0,0,0,0,-y_{7},-y_{6}, y_{5}, y_{4}\right)$, $\left(0,0,0,0,-y_{7},-y_{6}, y_{5}, y_{4}\right),\left(-y_{4},-y_{5},-y_{6}, y_{7}, 0,0,0,0\right),\left(-y_{5},-y_{4}, y_{7}, y_{6}, 0,0,0,0\right)$, $\left(-y_{6},-y_{7}, y_{4},-y_{5}, 0,0,0,0\right),\left(-y_{7}, y_{6},-y_{5},-y_{4}, 0,0,0,0\right)$.

While the last four vectors are normal to the three-sphere defined by the coordinates $\left\{\left(0,0,0,0, y_{4}, y_{5}, y_{6}, y_{7}\right) \mid y_{4}^{2}+y_{5}^{2}+y_{6}^{2}+y_{7}^{2}=1\right\}$, they can be viewed as tangent vectors to a distinct submanifold with coordinates $\left(y_{0}, y_{1}, y_{2}, y_{3}, 0,0,0,0\right)$. As the vectors are transported on the threesphere, the last three vectors trace a second three-sphere as tangents. Based on the isomorphism between $\left\{\left(y_{0}, y_{1}, y_{2}, y_{3}, 0,0,0,0\right) \mid y_{0}^{2}+y_{1}^{2}+y_{2}^{2}+y_{3}^{2}=1\right\}$ and $\left\{\left(0,0,0,0, y_{4}, y_{5}, y_{6}, y_{7}\right) \mid y_{4}^{2}+y_{5}^{2}+y_{6}^{2}+y_{7}^{2}=\right.$ 
$1\}$, a relabelling of the coordinates in the last four vectors gives

$$
\begin{aligned}
& -y_{0} \frac{\partial}{\partial y_{0}}-y_{1} \frac{\partial}{\partial y_{1}}-y_{2} \frac{\partial}{\partial y_{2}}+y_{3} \frac{\partial}{\partial y_{3}} \\
& -y_{1} \frac{\partial}{\partial y_{0}}-y_{0} \frac{\partial}{\partial y_{1}}+y_{3} \frac{\partial}{\partial y_{2}}+y_{2} \frac{\partial}{\partial y_{3}} \\
& -y_{2} \frac{\partial}{\partial y_{0}}-y_{3} \frac{\partial}{\partial y_{1}}+y_{0} \frac{\partial}{\partial y_{2}}-y_{1} \frac{\partial}{\partial y_{3}} \\
& -y_{3} \frac{\partial}{\partial y_{0}}+y_{2} \frac{\partial}{\partial y_{1}}-y_{1} \frac{\partial}{\partial y_{2}}-y_{0} \frac{\partial}{\partial y_{3}}
\end{aligned}
$$

At the point $\left(\begin{array}{llll}y_{0} & y_{1} & y_{2} & y_{3}\end{array}\right)$, the decomposition of vectors into tangent and normal components is

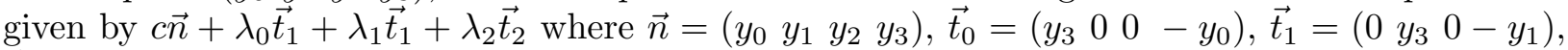
$\overrightarrow{t_{2}}=\left(\begin{array}{llll}0 & 0 & y_{3} & -y_{2}\end{array}\right)$, and for the first vector, the coefficients are $c=2 y_{3}^{2}-1, \lambda_{0}=-2 y_{0} y_{3}, \lambda_{1}=$ $-2 y_{0} y_{1}, \lambda_{2}=-2 y_{0} y_{2}$.

Six of the vector fields generate an $s u(2) \otimes s u(2)$ algebra. which is isomorphic to the antisymmetrized version of the colour algebra [43] over $\mathbb{R}$. The seventh vector field transforms nonlinearly under the action of one of the $\mathrm{SU}(2)$ groups.

The triality of the octonion algebra provides a theoretical basis for the mesons and baryons transforming as singlets under SU(3), lepton-hadron superselection rule with observables $\Omega$ being decomposible as a direct sum $\Omega=L \Omega L+H \Omega H, L H=H L=0, L+H=I d$ [43]. Together with unity 1 , the seven-dimensional colour algebra can be obtained from the octonion algebra through the direct sum $\mathbb{O}=F a \oplus W$, where $\mathrm{F}$ is the base field and $F 1+F a$ forms a subalgebra [39]. While the quark and anti-quark fields $\left\{u_{\alpha}, \bar{u}_{\alpha}\right\}$ can be regarded as triplets and anti-triplets of SU(3), the stability group for the seven-sphere $S U(4) / S U(3)$, their products $u_{\alpha} u_{\beta}=\epsilon_{\alpha \beta \gamma} \bar{u}_{\gamma}, \bar{u}_{\alpha} \bar{u}_{\beta}=\epsilon_{\alpha \beta \gamma} u_{\gamma}$ deine the colour algebra, together with the element $\mathrm{H}$ representing the hadron field [43].

The dependence of the coupling of a non-abelian gauge theory on the energy scale is given by the second Casimir invariant of the Lie group, which is determined by the structure constants $C_{2} \delta_{l m}=f_{l n n^{\prime}} f_{m n n^{\prime}}$,

$$
\frac{g^{2}}{g_{0}^{2}}=1+\left(11 C_{2}-2 n\right) \frac{g_{0}^{2}}{48 \pi^{2}} \ln (\Lambda L)^{2}+\mathcal{O}\left(g_{0}^{4}\right)
$$

where $g_{0}$ is the unrenormalized coupling, $\Lambda$ is the ultraviolet momentum cut-off and $L^{3}$ is the volume size. For an $\mathrm{SU}(3)$ gauge theory, with structure constants defined by $\left[\frac{1}{2} \lambda_{i}, \frac{1}{2} \lambda_{j}\right]=i f_{i j k} \lambda_{k}$, where $\lambda_{i}$ are the Gell-Mann matrices with normalization $\operatorname{tr}\left(\frac{1}{2} \lambda_{i} \frac{1}{2} \lambda_{j}\right)=\frac{1}{2} \delta_{i j}$ so that $f_{123}=1, f_{147}=$ $\frac{1}{2}, f_{246}=\frac{1}{2}, f_{257}=\frac{1}{2}, f_{345}=\frac{1}{2}, f_{516}=\frac{1}{2}, f_{637}=\frac{1}{2}, f_{458}=\frac{\sqrt{3}}{2}, f_{678}=\frac{\sqrt{3}}{2}$ and $C_{2}=3$. For the octonions, setting the normalization of the vector fields to be the same as that of the GellMann matrices, with $\left\langle\frac{1}{\sqrt{2}} e_{i}, \frac{1}{\sqrt{2}} e_{j}\right\rangle=\frac{1}{2} \delta_{i j}$ and $\left[\frac{1}{\sqrt{2}} e_{i}, \frac{1}{\sqrt{2}} e_{j}\right]=\frac{1}{\sqrt{2}} f_{i j k} \frac{1}{\sqrt{2}} e_{k} \equiv f_{i j k}^{\prime} \frac{1}{\sqrt{2}} e_{k}$, the identity $f^{\prime k m p} f^{\prime l n p}=\frac{1}{2} f^{k m p} f^{l n p}=\frac{1}{2}\left(\delta^{k l} \delta^{m n}-\delta^{k n} \delta^{m l}+\varphi^{k m l n}\right)$ implies that the equivalent quantity to $C_{2}$ also equals 3 and

$$
\frac{g^{2}}{g_{0}^{2}}=1+\left(11-\frac{2}{3} n\right) \frac{g_{0}^{2}}{8 \pi^{2}} \ln (\Lambda L)+\mathcal{O}\left(g_{0}^{4}\right)
$$

consistent with the energy-dependence of the strong-interaction coupling [44] This result suggests that all of the projected vector fields would be necessary for a description of strong interactions. 
The projections of the seven vector fields onto the tangent plane of the submanifold $S^{3} \times S^{3}=$ $\left\{\left(y_{0}, y_{1}, y_{2}, y_{3}, y_{4}, y_{5}, y_{6}, y_{7}\right) \mid y_{0}^{2}+y_{1}^{2}+y_{2}^{2}+y_{3}^{2}=\lambda, y_{4}^{2}+y_{5}^{2}+y_{6}^{2}+y_{7}^{2}=1-\lambda\right\}$ also can be computed. Given the six tangent vectors are

$$
\begin{aligned}
& \vec{t}_{0}=\left(1,0,0,-\frac{y_{0}}{y_{3}}, 0,0,0,0\right) \\
& \vec{t}_{1}=\left(0,1,0,-\frac{y_{1}}{y_{3}}, 0,0,0,0\right) \\
& \vec{t}_{2}=\left(0,0,1,-\frac{y_{2}}{y_{3}}, 0,0,0,0\right) \\
& \vec{t}_{4}=\left(0,0,0,0,1,0,0,-\frac{y_{4}}{y_{7}}\right) \\
& \vec{t}_{5}=\left(0,0,0,0,0,1,0,-\frac{y_{5}}{y_{7}}\right) \\
& \vec{t}_{6}=\left(0,0,0,0,0,0,1,-\frac{y_{6}}{y_{7}}\right)
\end{aligned}
$$

and the normal vector

$$
\vec{n}=\left(1, \frac{y_{1}}{y_{0}}, \frac{y_{2}}{y_{0}}, \frac{y_{3}}{y_{0}},-\frac{y_{4}}{y_{0}} \frac{\lambda}{1-\lambda},-\frac{y_{5}}{y_{0}} \frac{\lambda}{1-\lambda},-\frac{y_{6}}{y_{0}} \frac{\lambda}{1-\lambda},-\frac{y_{7}}{y_{0}} \frac{\lambda}{1-\lambda}\right)
$$

the equality between $c \vec{n}+\nu_{0} \vec{t}_{0}+\nu_{1} \vec{t}_{1}+\nu_{2} \vec{t}_{2}+\nu_{4} \vec{t}_{4}+\nu_{5} \vec{t}_{5}+\nu_{6} \vec{t}_{6}$ and each of the seven tangent vectors at $y$ can be used to determine the projection onto the tangent plane to the $S^{3} \times S^{3}$ submanifold. For the vector $\left(-y_{1}, y_{0}, y_{3},-y_{2}, y_{5},-y_{4}, y_{7},-y_{6}\right), c=0$, so that it lies in the tangent plane to the submanifold. The coefficient $c$ also vanishes for $\left(-y_{2},-y_{3}, y_{0}, y_{1}, y_{6},-y_{7},-y_{4}, y_{5}\right)$. However, for $\left(-y_{3}, y_{2},-y_{1}, y_{0},-y_{7},-y_{6}, y_{5}, y_{4}\right)$, the solution to the vector equality is

$$
\begin{aligned}
c & =(\lambda-1) y_{3} \\
\nu_{0} & =-\lambda y_{3} \\
\nu_{1} & =y_{2}+(1-\lambda) \frac{y_{1} y_{3}}{y_{0}} \\
\nu_{2} & =-y_{1}+(\lambda-1) \frac{y_{2} y_{3}}{y_{0}} \\
\nu_{4} & =y_{5}-\lambda \frac{y_{3} y_{4}}{y_{0}} \\
\nu_{5} & =-y_{4}-\lambda \frac{y_{3} y_{5}}{y_{0}} \\
\nu_{6} & =y_{7}-\lambda \frac{y_{3} y_{6}}{y_{0}}
\end{aligned}
$$

However, the condition $-\frac{\lambda}{1-\lambda} c \frac{y_{7}}{y_{0}}-\frac{\left(\nu_{4} y_{4}+\nu_{5} y_{5}+\nu_{6} y_{6}\right)}{y_{7}}=-y_{6}$ implies $-\lambda(1-\lambda) \frac{y_{3}}{y_{0} y_{7}}=0$, so that either $\lambda=0,1$ or $y_{3}=0$. Eliminating one of the three-spheres then reduces to the earlier choice of the three-sphere submanifold. If $0<\lambda<1$, the projected vector is $\left(0, y_{2},-y_{1}, \lambda y_{0}, y_{5},-y_{4}, y_{7},-y_{6}\right)$. 
Given the vector $\left(-y_{4},-y_{5},-y_{6}, y_{7}, y_{0}, y_{1}, y_{2},-y_{3}\right)$, the equality implies that

$$
\begin{aligned}
c & =-\nu_{0}-y_{4}=-\frac{1}{\lambda}\left[\frac{y_{1} y_{5}}{y_{0}}+\frac{y_{2} y_{3}}{y_{0}}-y_{4} \frac{\left(\lambda-y_{0}^{2}\right)}{y_{0} y_{3}}-y_{7}\right]-y_{4} \\
\nu_{0} & =\frac{1}{\lambda}\left[\frac{y_{1} y_{5}}{y_{0}}+\frac{y_{2} y_{3}}{y_{0}}-y_{4} \frac{\left(\lambda-y_{0}^{2}\right)}{y_{0} y_{3}}-y_{7}\right] \\
\nu_{1} & =\frac{1}{\lambda} \frac{y_{1}}{y_{0}}\left[\frac{y_{1} y_{5}}{y_{0}}+\frac{y_{2} y_{3}}{y_{0}}-y_{4} \frac{\left(\lambda-y_{0}^{2}\right)}{y_{0} y_{3}}-y_{7}\right]+\frac{\left(y_{1} y_{4}+y_{0} y_{5}\right)}{y_{0}} \\
\nu_{2} & =\frac{1}{\lambda} \frac{y_{2}}{y_{0}}\left[\frac{y_{1} y_{5}}{y_{0}}+\frac{y_{2} y_{3}}{y_{0}}-y_{4} \frac{\left(\lambda-y_{0}^{2}\right)}{y_{0} y_{3}}-y_{7}\right]+\frac{\left(y_{2} y_{4}+y_{0} y_{3}\right)}{y_{0}} \\
\nu_{4} & =y_{0}-\frac{y_{4}}{y_{0}} \frac{1}{1-\lambda}\left(\frac{y_{1} y_{5}}{y_{0}}+\frac{y_{2} y_{3}}{y_{0}}-y_{7}-\frac{y_{4}\left(\lambda-y_{0}^{2}\right)}{y_{0} y_{3}}+\lambda y_{4}\right) \\
\nu_{5} & =y_{1}-\frac{y_{5}}{y_{0}} \frac{1}{1-\lambda}\left(\frac{y_{1} y_{5}}{y_{0}}+\frac{y_{2} y_{3}}{y_{0}}-y_{7}-\frac{y_{4}\left(\lambda-y_{0}^{2}\right)}{y_{0} y_{3}}+\lambda y_{4}\right) \\
\nu_{6} & =-\frac{1}{1-\lambda} \frac{y_{7}}{y_{6}}\left(\frac{y_{1} y_{5}}{y_{0}}+\frac{y_{2} y_{3}}{y_{0}}-y_{7}-\frac{y_{4}\left(\lambda-y_{0}^{2}\right)}{\left.y_{0} y_{3}\right)+\frac{y_{4} y_{7}^{2}}{y_{0} y_{6}}}\right. \\
& +\frac{y_{4}^{2}}{y_{6} y_{0}}\left(y_{0}-\frac{1}{1-\lambda}\left(\frac{y_{1} y_{5}}{y_{0}}+\frac{y_{2} y_{3}}{y_{0}}-y_{7} \frac{y_{4}\left(\lambda-y_{0}^{2}\right)}{y_{0} y_{3}}\right)+\lambda y_{4}\right) \\
& +\frac{y_{5}^{2}}{y_{6} y_{0}}\left(y_{1}-\frac{1}{1-\lambda}\left(\frac{y_{1} y_{5}}{y_{0}}+\frac{y_{2} y_{3}}{y_{0}}-y_{7} \frac{y_{4}\left(\lambda-y_{0}^{2}\right)}{y_{0} y_{3}}\right)+\lambda y_{4}\right)
\end{aligned}
$$

indicating the nonlinear nature of the components of the last four projected vectors.

\section{Dimensional Reduction over Coset Manifolds and Residual Gauge Symmetry}

The action of generalized gauge transformations on bundles with an $S^{7}$ fibre, and the residual gauge symmetries, have been studied in $\S 3$. These results can be compared with the dimensional reduction of 11-dimensional supergravity over $M_{4} \times S^{7}$ [45] and dimensional reduction of superstring theory from ten to four dimensions [46].

Given a coset manifold $S / R$, dimensional reduction of the theory can be achieved automatically when all of the fields are required to be invariant under the group S. For a tensor field, this implies that

$$
T^{\mu_{1} . . \mu_{n}}(g(x, y))=\frac{\partial g(x, y)^{\mu_{1}}}{\partial X^{\rho_{1}}} \ldots \frac{\partial g(x, y)^{\mu_{n}}}{\partial X^{\rho_{n}}} T^{\rho_{1} \ldots \rho_{n}}(x, y) \quad X^{\mu}=(x, y)
$$

It has been shown that S-invariance of a field on $M_{4} \times S / R$ follows from R-invariance of the field at a designated base point [45] of the homogeneous coset manifold.

In contrast to fields which transform covariantly with respect to space-time symmetry transformations, symmetric gauge fields do not have to be strictly invariant but only satisfy the weaker condition that the transformed field is gauge-equivalent.

When the coset manifold is $S^{7}$, and $\mathrm{S}$ is the group $\mathrm{SU}(4)$, then $\mathrm{SU}(4)$ invariance of a vector 
field $V^{\alpha}(x, y)$,

$$
V^{\alpha}(x, g(y))=\frac{\partial(g(y))^{\alpha}}{\partial y^{\gamma}} V^{\gamma}(x, y)
$$

is equivalent to $\mathrm{SU}(3)$ invariance at the base point

$$
V^{\alpha}(x, o)=\left.\frac{\partial(h(y))^{\alpha}}{\partial y^{\gamma}}\right|_{y=o} V^{\gamma}(x, o)
$$

where $h \in R$, the stabilizing group of the origin o. Consequently, the only unconstrained fields over the base space $M_{4}$ are the $\mathrm{SU}(3)$ singlets.

In this case, the decomposition of the adjoint representation of $\mathrm{S}$ into irreducible representations of $\mathrm{R}$ provides the gauge groups of the dimensionally reduced field theory. When $S=S O(8)$, $\underline{28} \rightarrow \underline{21}+\underline{7}$ and when $S=S U(4), \underline{15} \rightarrow \underline{8}+\underline{3}+\underline{\overline{3}}+\underline{1}$. The number of unconstrained gauge potentials is given by the number of R-singlets in the isotropy representation $I_{R}$, or equivalently, the dimension of $\mathrm{C}(\mathrm{R})$, the centralizer of $\mathrm{R}$ in $\mathrm{S}$. Consequently, dimensional reduction of an Sinvariant gauge theory in $M_{4} \times S / R$ should give a theory with unconstrained gauge potentials on $M_{4}$ transforming under the symmetry group $C(R)$. When $S=S O(8)$, this residual symmetry group only consists of the identity element, whereas, when $S=S U(4)$, the symmetry group is U(1) [43]. These results are consistent with those obtained through the calculation of the transformation of the connection form in $\S 3$.

In a modification of this technique, S-invariance can be extended to symmetric gauge fields which satisfy a generalized gauge invariance law [47]

$$
\begin{aligned}
g(s, x) A_{\rho} g^{-1}(s, x)+\partial_{\rho} g(s, x) g^{-1}(s, x)=A_{\mu}(s(x)) & J_{\rho}^{\mu}(s, x) \\
s & \in S, g(s, x) \in G
\end{aligned}
$$

indicating that the potential is invariant up to a gauge transformation. If the initial gauge group is $G$, and $K(R)$ is the homomorphic image of $R$ in $G$, then after dimensional reduction, the gauge group is the centralizer in $\mathrm{G}$ of $\mathrm{K}(\mathrm{R})$ [45]. For different choices of $\mathrm{G}$, the use of symmetric gauge fields leads to a wider variety of dimensional reduction schemes [47][48] and therefore might be used to obtain larger residual gauge groups beginning with an invariance under $S=S U(4)$. In particular, the residual symmetry group perhaps can be enlarged to the stability group $H$ for the coset space $\mathrm{G} / \mathrm{H}$, thus obtaining the full set of linear gauge transformations [49] when $\mathrm{G}$ is nonlinearly realized.

Solutions to the $d=11$ supergravity equations of motion with SU(4) symmetry, include, for example, an $S^{7}$ metric obtained by stretching U(1) fibres over $\mathbb{C P}^{3}$ [50][51] The higher-dimensional action may consist of terms containing fields transforming under different irreducible representations of $\mathrm{SU}(3)$, and integration over the fibre coordinates can be performed, giving rise to an action on $M_{4}$. Specifically, when $S=S U(4)$ and $R=S U(3)$, amongst the non-trivial representations in the decomposition of ad $S U(4)$ are those corresponding to the $\mathrm{SU}(3)$ gauge field, which could also be included in the dimensionally reduced action. This would therefore providing a method for obtaining the QCD gauge theory for strong interactions in four dimensions from a higher-dimensional theory through the geometrical procedure of reduction over a coset space.

In addition to this method for obtaining an $\mathrm{SU}(3)$ gauge theory, results of a systematic study of Lagrangians containing fields transforming linearly under a group $\mathrm{R}$ but nonlinearly under a larger 
group S [52][53] could be used in this case. Since it is known that a theory with fields transforming linearly under $\mathrm{R}$ can be shown to be equivalent to a theory with fields transforming nonlinearly under $\mathrm{S}$, pure gauge fields on $\mathrm{S} / \mathrm{R}$ may be added to gauge fields on $\mathrm{R}$ to obtain a Lagrangian with local gauge invariance under $\mathrm{S}$ [54]. Given an element of the coset space $\phi_{0}(x) \in S / R$, and gauge fields in the Lie algebra of $\mathrm{R}, A_{\mu} \in \mathcal{R}$, the pure gauge fields [54] are defined to be

$$
B_{\mu}=\phi_{0}(x)\left(\partial_{\mu}+A_{\mu}\right) \phi_{0}^{-1}(x)
$$

and the set $\left\{A_{\mu}, B_{\mu}\right\}$ forms a nonlinear representation of $\mathrm{S}$ and the field content of an S-invariant Lagrangian. It may be noted that the extra fields are derived from scalar quantities and therefore resemble the coordinate fields of a higher-dimensional theory. Within the context of the nonlinear realization approach, the physical equivalence of the R-invariant and S-invariant theories follows from the elimination of the pure gauge fields by gauge transformations, whereas the dimensional reduction of a higher-dimensional theory produces a closely related but nevertheless distinct theory. The usefulness of pure gauge fields on coset spaces depends on whether the spin-one gauge field in the ten-dimensional action possesses $\mathrm{SU}(4)$ symmetry or an $\mathrm{SU}(3)$ symmetry that is being viewed as $\mathrm{SU}(4)$ through the method of induced representations.

Although reduction over $S^{7}=S U(4) / S U(3)$ has been considered exclusively thus far in this section, it may be noted that compactification of ten-dimensional superstring theories on sixdimensional compact spaces is also known to lead to a breaking of an SU(4) symmetry through an SU(3) subgroup. For example, one may consider the reduction of ten-dimensional supergravity to four dimensions when all of the fields are independent of the extra six coordinates $y^{\alpha}$. The resulting $\mathrm{N}=4$ supersymmetry is generated by 4 spinors $Q^{A}$ transforming under the fundamental representation of SU(4). As they also transform as $1 \oplus 3$ under an $\mathrm{SU}(3)$ subgroup of $\mathrm{SU}(4)$, this invariance under this subgroup breaks the $N=4$ supersymmetry to $N=1$, with the surviving supersymmetry being an $\mathrm{SU}(3)$ singlet [46]. Similar considerations apply to the breaking of $E_{8}$ to $E_{6}$ or grand unification, in the compactification of ten-dimensional $E_{8} \times E_{8}$ supergravity theories on Calabi-Yau manifolds with SU(3) holonomy.

The free Green-Schwarz superstring can be formulated in three, four and six dimensions and a Lorentz covariant and unitary interacting Green-Schwarz superstring exists in ten dimensions [55]. These dimensions are necessary for local supersymmetry, which depends on $\Gamma$-matrix identities derived from the division algebras $\mathbb{R}, \mathbb{C}, \mathbb{H}$ and $\mathbb{O}[56]$ corresponding to the transverse directions. Classical solutions of the equations of motion of the Green-Schwarz Lagrangian in $D=10$ have been found by expressing ten-dimensional vectors as $2 \times 2$ octonionic matrices and 32-real-component Majorana spinors as spinors with four octonionic components [57]. These equivalences follow from the isomorphism $\tilde{S O}(1,9) \simeq S L(2, \mathbb{O})$ [58][59] which is the last in a sequence of isomorphisms involving space-time and division algebras, $\tilde{S O}(1, \nu+1) \simeq S L\left(2 ; \mathbb{K}_{\nu}\right), \nu=1,2,4,8[60]$.

These isomorphisms can be used in the representation of space-time vectors as $2 \times 2$ hermitian matrices and null vectors as fermion bilinears [60]. The null vector $P^{\mu}$ is equivalent to

$$
P=\lambda \lambda^{\dagger}=\left(\begin{array}{cc}
\xi \xi^{\dagger} & \xi \eta^{\dagger} \\
\eta \xi^{\dagger} & \eta \eta^{\dagger}
\end{array}\right)
$$

where $\lambda=\left(\begin{array}{l}\xi \\ \eta\end{array}\right)$. Since the determinant of this matrix, which equals $P^{\mu} P_{\mu}$, vanishes, $P^{\mu}$ must lie on the forward light cone. Lorentz transformations on the space-time vector $P^{\mu}$ can be regarded as $S L\left(2, \mathbb{K}_{\nu}\right)$ transformations on $\lambda \lambda^{\dagger}$ derived from the multiplication of $S L\left(2, \mathbb{K}_{\nu}\right)$ matrices and the spinor $\lambda[34]$. 
The space of light-like lines at a point in ten dimensions is $S^{8}$ and the above equivalence implies that it can be represented as the set of spinors $\lambda$ modulo transformations which leave $\lambda \lambda^{\dagger}$ invariant. These transformations form the algebra $S^{7}$ and the action on the space of light-like lines is given by the Hopf fibration $S^{15} \rightarrow S^{8}$ [60]. This construction has also been extended to the action of $S^{7}$ on the physical twistor space $\mathcal{N} \subset \mathbb{O} P^{3}[61]$ and the $S^{7}$ Kac-Moody algebra $\hat{S}^{7}[62]$ which arises as a symmetry algebra of the twistor-string theory [63] and the light-cone superstring [64]. Finally, supersymmetrical light-like lines have been used in ten-dimensional super-Yang-Mills theories to integrate the constraint equations [65] and an $S^{7}$ symmetry on the space of light-like lines exists.

A solution to the problem of the fibre coordinate dependence of the transformation rule of the connection form for a general bundle implies that a Lie group structure is necessary for a pure gauge theory. While the projection of vector fields on the seven-sphere to a group submanifold appears to provide an exception as it can be used to introduce a potential with seven non-zero components, an underlying $S U(2) \times S U(2)$ group structure is necessary to define gauge transformations of six of the these components.

The problem of force unification can be refined by the classification of matter multiplets, since the components of the spinors must take values in the division algebras. Basing the fermionic part of the standard model on the spinor space $T=\mathbb{C} \otimes \mathbb{H} \otimes \mathbb{O}$, it may be noted that the amplitudes for elementary particle interactions typically involve the product of two fermions $\psi_{1}, \psi_{2}$ and a vector boson $A^{\mu}$ at the vertices of the perturbative diagrams. If the fermions did take values in a composition algebra with zero divisors, this vertex factor could vanish because there then would exist $\psi_{1}, \psi_{2} \neq 0$ such that $\psi_{1} \cdot \psi-2 \neq 0$.

The sequence of division algebras can be extended to higher-dimensional Cayley-Dickson algebras containing zero divisors $u_{l}, u_{m}$, such that their product vanishes [66]. It follows that since $\left(u_{k} u_{l}\right) u_{m}=u_{k}\left(u_{l} u_{m}\right)+\left\{u_{k}, u_{l}, u_{m}\right\}$ this expression vanishes if the associator $\left\{u_{k}, u_{l}, u_{m}\right\}$ is zero. For Cayley-Dickson algebras of dimension $2^{n}, n \geq 4$, there are triples of basis elements for which $\left\{e_{k}, e_{l}, e_{m}\right\}=0$. Since $u_{l}$ is a linear combination of the basis elements $\sum_{i} c_{i} e_{i}$, a rotation of the basis elements, given by $e_{i}^{\prime}=\frac{1}{\sum_{j} c_{j}^{2}} \sum_{i} c_{i} e_{i}$, is sufficient to generate a zero divisor. In fact, the dimension of the kernel of the operator $L_{a}$, representing left multiplication by a zero divisor $a$, satisfies the condition $2^{n}-4 \geq \operatorname{dim} \operatorname{ker} L_{a} \equiv 0(\bmod 4)$ [66]. The same elements of a Cayley- Dickson algebra will represent $k e r R_{a}$. Since it can be verified that the zero-divisors in the Cayley-Dickson algebras are combinations of the basis elements, one may apply a transformation to the standard basis $\left\{e_{0}, e_{1}, e_{2}, \ldots, e_{2^{n}-1}\right\}$ such that the new basis include the zero divisors. The multiplication table will then have block form so that the fermion terms in the Lagrangian and the corresponding vertex factors will vanish. Since the blocks will be $4 \times 4$, it should possible to express the particle content again in terms of sums of tensor products of spinor spaces which are isomorphic to $\mathbb{C}, \mathbb{H}$ and $\mathbb{O}$. The overall symmetry of the action would also be reduced according to the requirement that block form be maintained.

In addition to the finite-dimensional Clifford algebra, $\mathbb{C}_{L} \times \mathbb{H}_{L} \times \mathbb{O}_{L}=R_{0,9}$, infinite-dimensional Clifford algebras, in principle, might be used to describe elementary particle interactions. Consider a set of unitary operators $U_{i}: R^{n} \rightarrow R^{n}$ such that $U_{i}^{2}=-I$ and $U_{i} U_{j}=-U_{j} U_{i}$. Let $n=$ $(2 a(n)+1) 2^{b(n)}, b(n)=c(n)+4 d(n), 0 \leq c(n) \leq 3$. The maximal number of unitary operators on $R^{n}$ satisfying the Clifford algebra relations, is equal to $\rho(n)-1$, where $\rho(n)=2^{c(n)}+8 d(n)$ by the Hurwitz-Radon-Eckmann theorem [67][68][69]. When $n$ is finite, it is known that $\rho(n) \leq n$ and that $\rho(n)=n$ only for $n=1,2,4,8$. Moreover, $\rho(\infty)=\infty$, so that the infinite-dimensional Clifford 
algebra necessarily acts on an infinite-dimensional vector space. It may be noted that $\rho(n)-1$ also represents the maximal number of linearly independent vector fields on $S^{n-1}$, replacing the products of basis operators $U_{i}$ by Lie brackets of unit vector fields on the sphere, $X_{i}$. As an infinite-dimensional division algebra can be viewed as a module for the Clifford algebra, no finitedimensional representation can be used for a spinor space. Since there are only a finite number of fermion degrees of freedom in a realistic unified field theory, infinite-dimensional division algebras would not be relevant in the formulation of this theory.

The vector boson, the carrier of the force, is initially massless when the gauge symmetry is unbroken and the expression of its momentum vector as a spinor bilinear in ten dimensions is consistent with the diagram representing the interaction between the gauge field and the fermions. Since the potential belongs to the adjoint representation of the gauge group, the symmetry groups of the theories describing the elementary particle interactions then would be determined by the fermions. The restriction of the fermions to the division algebras should then provide a theoretical principle for explaining the types of gauge groups that appear in the standard model. The groups which act most naturally on $T=\mathbb{C} \otimes \mathbb{H} \otimes \mathbb{O}$ are those which are subgroups of the adjoint left algebra $T_{L}(2) \sim \mathbb{C}(32)$ or the Clifford algebra $R_{1,9}$, specifically those groups generated by the subspace of two-vectors of $R_{1,9}, \operatorname{so}(1,9), \mathbb{O}_{L}, s o(6) \sim s u(4)$, and $\mathbb{C}_{L} \otimes \mathbb{H}_{L}, \operatorname{su}(2)$.

Values of the charges of fermions transforming under $U(1)$, the isospin group $S U(2)$ and the global flavour group $S U\left(N_{f}\right)$ are integers or multiples of an elementary fraction in the standard model, and the integral elements of the complex numbers, quaternions and octonions, constructed from \pm 1 , the real integers and the half integers $\pm \frac{1}{2}$ [70] may be used in the root lattices arising in chiral fermionic strings [71]. However, the vertex wave functions that arise in the perturbative diagrams of the S-matrix expansion should be based on a field of complex numbers, consistent with the fundamental axioms of quantum mechanics. Non-trivial interactions between fermions belonging to selected sets of multiplets may require the spinor space to be restricted to the form $\mathbb{C}^{n_{\mathbb{C}}} \otimes \mathbb{H}^{n_{\mathbb{H}}} \otimes \mathbb{O}^{n_{\mathbb{Q}}}$. The vanishing of the product $\left(z_{1}, 0\right) \cdot\left(0, z_{2}\right)$ in $\mathbb{C}^{2}$ implies the existence of zero divisors when $n_{\mathbb{C}} \geq 2$, and it has been proven that the tensor product of two rational generalized quaternion division algebras is not a division algebra [72], so that zero divisors in the spinor space arise when $n_{\mathbb{H}} \geq 2$. This theorem can be extended immediately to the tensor product of octonion algebras.

The spinor space $T$ defined by $n_{\mathbb{C}}=n_{\mathbb{H}}=n_{\mathbb{O}}=1$ encompasses only one generation of fermions. The choice of $n_{\mathbb{C}}=n_{\mathbb{H}}=n_{\mathbb{O}}=3$ would imply that there are interactions between the three different generations of fermions in the standard model. However, this does not occur without the mediation of a vector boson, which splits the interaction diagram into two parts, each representing a different sector of the spinor space. At each vertex, the interaction between the fermions of each generation and the vector boson are represented by a non-trivial amplitude factor, as the vector boson can be allowed to interact universally, but there is no direct interaction between the fermions of different generations at a single vertex. It is preferable therefore to regard the spinor space for the standard model as the direct sum of three copies of the $\mathbb{C} \otimes \mathbb{H} \otimes \mathbb{O}$ rather than tensor product $\mathbb{C}^{3} \otimes \mathbb{H}^{3} \otimes \mathbb{O}^{3}$. Furthermore, the latter spinor space contains zero divisors, so that the condition of the non-existence of zero divisors could not be directly used to restrict the sets of fermions and consequently the gauge groups in the standard model. While $\oplus_{i=1}^{3} \mathbb{C}_{i} \otimes \mathbb{H}_{i} \otimes \mathbb{O}_{i}$ is a subspace of $\mathbb{C}^{3} \otimes \mathbb{H}^{3} \otimes \mathbb{O}^{3}$, the algebraic composition is applied only to the spinor space $\mathbb{C}_{i} \otimes \mathbb{H}_{i} \otimes B b b O_{i}$ for each $i$. Consequently, use of the direct sum of the three copies is consistent with the restriction on the fermion space defined by the principle of non-existence of zero divisors. 
It has been established through the Hurwitz theorem that division algebras over the real numbers only have dimension $1,2,4,8$, and a classification of four-dimensional real quadratic algebras [73] has been obtained, together with a new eight-dimensional quadratic division algebra. A preference for the modules $\mathbb{C}, \mathbb{H}$ and $\mathbb{O}$ is based on the equivalence of the conventional space-time symmetry groups and gauge groups with the groups of linear transformations acting on these spaces and their proper subgroups. Specifically, the existence of a norm these division algebras induces the symmetry groups required for the construction of the gauge theories. The number of generations of fermions, and therefore the number of copies of $\mathbb{C} \otimes \mathbb{H} \otimes \mathbb{O}$, is known to be constrained by the cancellation of anomalies [74], suggesting an essentially unique spinor space for the standard model.

\section{Conclusion}

It has been shown that elimination of the fibre coordinate in the transformation rule of the connection form in bundles with a structure group larger than the standard fibre, and in particular for bundles with the structure group given the isometry group of the standard fibre, leads to restrictions on the bundle. For the $S^{3}$ bundle, the allowed group of gauge transformations is reduced from the isometry group $\mathrm{SO}(4)$ to $\mathrm{SU}(2)$. For the $S^{7}$ bundle, not all of the conditions deriving from fibre-coordinate independence can be satisfied, so that the transformation rules retain a dependence on the fibre coordinate. The requirement of independence with respect to the coordinates of the entire $S^{7}$ fibre leaves no residual gauge symmetry beginning with an $\mathrm{SO}(8)$ structure group and only a U(1) symmetry starting with an SU(4) structure group. Similar conditions can be placed on the structure group and gauge transformations in quantum principal bundles. It is established that a Lie group structure is required for the pure gauge theory and that any application of the division algebras to force unification must be restricted initially to the organization of the fermion multiplets in the standard model. Nevertheless, this suggests a theoretical principle which distinguishes the specific gauge groups that do arise in theories of elementary particle interactions. It has been noted that the fermion part of the standard model can be based on the spinor space $T=\mathbb{C} \otimes \mathbb{H} \otimes \mathbb{O}$, and the necessity of the division algebras in the organization of the fermion mutiplets is explained. Amplitudes for elementary particle interactions typically involve the product of two fermions $\psi_{1}, \psi_{2}$ and a vector boson $A^{\mu}$ at the vertices of perturbative diagrams, and their non-vanishing follows follow directly from the fermions taking values in the division algebras. Since the unified theory is initially formulated in ten dimensions, the masslessness of the vector boson, when the gauge symmetry is unbroken, implies that the momentum vector can be expressed as a spinor bilinear. Relating the gauge potential itself to a spinor bilinear, the symmetry groups of the theories describing elementary particle interactions then would be determined by the restriction of the fermions to the division algebras. As the exchange of intermediate string states describes the exchange of vector bosons in the field theory limit, this approach points toward a connection between the geometry of the internal symmetry spaces arising in the standard model and superstring theory.

\section{Acknowledgements}

I would like to thank Prof. Elmar Schrohe for his hospitality while this work has been completed at the Institut für Mathematik, Universität Potsdam. Useful conversations with Dr. G. M. Dixon regarding gauge theories and the seven-sphere and Dr. D. Colferai about experimental tests of quantum chromodynamics are gratefully acknowledged. I thank Mr Allan Steel for providing the Magma programme to compute the matrix terms in the transformation rule of the connection of a 
sub-bundle of the $S^{7}$ bundle. Financial support has been received from Alexander von Humboldt Foundation during the completion of the investigation. 


\section{Appendix}

\section{A. Independence of the Transformation of the Connection Form with respect to the Fibre Coordinate}

For a general fibre bundle with atlas $\left\{U_{\alpha}, \psi_{\alpha}\right\}$, the trivializations $\psi_{\alpha}, \psi_{\beta}$ determine two local sections $\sigma_{\alpha}(x)=\psi_{\alpha}^{-1}\left(x, y_{0}\right), \sigma_{\beta}(x)=\psi_{\beta}^{-1}\left(x, y_{0}\right)$ which may be mapped by a diffeomorphism to $(x, y(x))$ and $\left(x, y^{\prime}(x)\right)$. If $\psi_{\beta \alpha}: U \times F \rightarrow F$, where $U \subset U_{\alpha} \cap U_{\beta}$, is defined by $\psi_{\beta \alpha}(x, y)=y^{\prime}$, then the tangent spaces to the two sections are given by $\left(\xi_{x}, V^{\alpha}\right)$ and $\left(\xi_{x}, T \psi_{\beta \alpha}\left(\xi_{x}, V^{\alpha}\right)\right)$. A connection provides a splitting of the tangent bundle into vertical and horizontal sub-bundles, and the image of the horizontal subspaces associated with the two sections in $T U \times T F$ are spanned by vectors of the form $\left(\xi_{x}, C^{\alpha}(y)\right)$ and $\left(\xi_{x}, C^{\beta}\left(y^{\prime}\right)\right)$. Defining the connection forms $\Gamma^{\alpha}$ by $V^{\alpha}-C^{\alpha}$, one finds that they transform under a change of section as

$$
\Gamma^{\beta}\left(\xi_{x}, y^{\prime}\right)=T \psi_{\beta \alpha}^{y}\left(\xi_{x}\right)+T \psi_{\beta \alpha}^{x} \cdot \Gamma^{\alpha}\left(\xi_{x}, y\right)
$$

where $\psi_{\beta \alpha}^{x}(y) \equiv \psi_{\beta \alpha}^{y}(x)=y^{\prime}$.

The tangent mapping $T \psi_{\beta \alpha}^{x}: T F \rightarrow T F$ is an isomorphism if the tangent bundle of the fibre is trivializable. Defining $\phi_{y}$ to be a mapping from a vector space $\mathrm{V}$ to the tangent space $T_{y}(F)$, so that $\Gamma^{\alpha}\left(\xi_{x}, y\right)=\phi_{y} \Gamma^{\alpha}\left(\xi_{x}\right), \Gamma^{\alpha}\left(\xi_{x}\right) \in V$, equation (A1) becomes

$$
\phi_{y^{\prime}} \Gamma^{\beta}\left(\xi_{x}\right)=T \psi_{\beta \alpha}^{y}\left(\xi_{x}\right)+T \psi_{\beta \alpha}^{x} \cdot \phi_{y} \Gamma^{\alpha}\left(\xi_{x}\right)
$$

To interpret the relation between $\Gamma^{\alpha}\left(\xi_{x}\right)$ and $\Gamma^{\beta}\left(\xi_{x}\right)$ as a gauge transformation of potentials taking values on the base space, it is necessary to eliminate the fibre coordinate dependence. This can be achieved if the right-hand side of the equation can be expressed as $\phi_{y^{\prime}} X, X \in V$ and $\phi_{y}$ is an injective mapping. Moreover, $\mathrm{V}$ can be extended to have the same dimension as $\mathrm{F}$, so that $\Gamma^{\alpha}\left(\xi_{x}, y\right)$ ranges over all of $T_{y}(F)$ and $\phi_{y}$ is a surjective mapping. Thus $\phi_{y}$ should be a bijection be a bijection. Writing $T \psi_{\beta \alpha}^{y}\left(\xi_{x}\right)$ as $\phi_{y^{\prime}} X_{1}, X_{1} \in V$ and $T \psi_{\beta \alpha}^{x} \cdot \phi_{y} \Gamma^{\alpha}\left(\xi_{x}\right)$ as $\phi_{y^{\prime}} X_{2}, X_{2} \in V$, a fibre coordinate-independent gauge transformation rule may be obtained if

$$
\phi_{y^{\prime}}\left(X_{1}\right)+\phi_{y^{\prime}}\left(X_{2}\right)=\phi_{y^{\prime}}\left(X_{1}+X_{2}\right)
$$

expressing linearity of $\phi_{y}$. It may be noted that a bijective mapping between the vector spaces that is nonlinear, analogous to the mapping $x \rightarrow x^{3}$ on $(\mathbb{R},+)$, could also have been considered. As $\Gamma^{\alpha}\left(\xi_{x}, \cdot\right)$ is a $C^{r}, r>1$ vector field on $\mathrm{F}$, the mapping $\phi: V \times F \rightarrow T F, \phi(\cdot, y)=\phi_{y}$ must be a differentiable function of the fibre coordinate. Moreover, $\phi_{y}$ should also be a vector space isomorphism, since one takes $\Gamma^{\alpha}\left(\xi_{x}, y\right)+\Gamma^{\alpha}\left(\eta_{x}, y\right)$ to be $\Gamma^{\alpha}\left(\xi_{x}+\eta_{x}, y\right)$ as a result of the trivializations $\left\{\psi_{\alpha}\right\}, \psi_{\alpha}: \pi^{-1}\left(U_{\alpha}\right) \rightarrow U_{\alpha} \times F$ being diffeomorphisms. The requirement that $\phi_{y}$ be a vector space isomorphism would exclude nonlinear mappings and ensure that equation (A2) can be used in reducing the transformation rule of the connection form to a gauge transformation involving a dependence on the base space coordinates only.

The definitions of several types of fibre parallelisms are given [75]:

\section{Definition A1. Fibre parallelism}

There exists a map $C: E \times_{M} E \rightarrow \underset{\left(y, y^{\prime}\right) \in E \times_{M} E}{\cup} I \operatorname{som}\left(T_{y} E, T_{y^{\prime}} E\right)$ such that $C\left(y, y^{\prime}\right)=\omega_{y^{\prime}} \circ \omega_{y}^{-1}$ : $T_{y} E \rightarrow T_{y^{\prime}} E$ where $y, y^{\prime} \in E_{x}$ and $\omega_{y}:\left(T_{r e d} E\right)_{x} \rightarrow T_{y} E$. The last map gives rise to an isomorphism $\Omega: T_{\text {red }} E \times_{M} E \rightarrow T E$. 


\section{Definition A2. Vertical fibre parallelism}

There exists a map $\tilde{C}: E \times_{M} E \rightarrow \underset{\left(y, y^{\prime}\right) \in E \times_{M} E}{\cup} \operatorname{Isom}\left(T_{y} E_{x}, T_{y^{\prime}} E_{x}\right)$ such that $\tilde{C}\left(y, y^{\prime}\right): T_{y} E_{x}=$ $V_{y} E \rightarrow T_{y^{\prime}} E_{x}=V_{y^{\prime}} E$. This parallelism is given by the isomorphism $\tilde{\Omega}: V T_{\text {red }} E \times_{M} E \rightarrow V T E$.

\section{Definition A3. Integrable fibre parallelism}

A fibre parallelism is integrable if, for any $\left(y, y^{\prime}\right) \in E \times{ }_{M} E$, there exists a translation $\tau_{y^{\prime} y}: E \rightarrow$ $E, \tau_{y^{\prime} y}(y)=y^{\prime}$ such that $T \tau_{y^{\prime} y} \in C\left(E \times_{M} E\right)$. For a vertical fibre parallelism, there exists a translation $\tau_{y^{\prime} y}^{\text {vert }}: E_{x} \rightarrow E_{x}$ such that $T \tau_{y^{\prime} y}^{v e r t} \in \tilde{C}\left(E \times_{M} E\right)$. It may be noted that a surmersion $(E, M, \pi)$ that admits a vertical fibre parallelism and a connection is a projectable fibre parallelism. [If $(E, M, \pi)$ is a fibration, then a vertical fibre parallelism implies the existence of a connection.] A projectable fibre parallelism with an integrable parallelism on each fibre, or equivalently such that the commutator of invariant vertical vector fields is invariant, can also be regarded as an integrable vertical fibre parallelism.

\section{Definition A4. Globally integrable fibre parallelism}

It can be shown that a fibration which admits a globally integrable fibre parallelism is a principal bundle. The translation $\tau_{y^{\prime} y}: E \rightarrow E$ can be extended to a global diffeomorphism on E.

For a principal bundle,

$$
\begin{aligned}
V T P & =T_{e}(G) \times P=V T P / G \times{ }_{M} P=V T_{\text {red }} E \times{ }_{M} P \\
V T P / G & =\{\text { right }- \text { invariant vector fields tangent to the fibres of } E\} \\
V T_{\text {red }} E & \sim T_{e}(G) \times M
\end{aligned}
$$

$V T P / G$ is clearly a trivial bundle, because $(V T P / G)_{x} \sim T_{e}(G) \forall x \in M$, and the transition functions are elements of $\mathrm{G}$, leaving invariant any vector field in $V T P / G$.

The parallelism in a principal bundle can be taken to be the one induced by left multiplication and the diffeomoerphism $\psi_{\beta \alpha}^{x}$ to be right multiplication by a group element. Since

$$
\begin{aligned}
R_{g *} L_{y *} & =L_{(y \cdot g) *} A d\left(g^{-1}\right) \\
T \psi_{\beta \alpha}^{y}\left(\xi_{x}\right) & =L_{y *}\left(g_{*} \cdot \xi_{x}\right)=L_{y \cdot g *}\left[L_{g *}^{-1}\left(g_{*} \cdot \xi_{x}\right)\right]=\phi_{y^{\prime}}\left[L_{g *}^{-1}\left(g * \cdot \xi_{x}\right)\right]
\end{aligned}
$$

the standard gauge transformation

$$
\Gamma^{\beta}\left(\xi_{x}\right)=A d\left(g^{-1}\right) \Gamma^{\alpha}\left(\xi_{x}\right)+L_{g *}^{-1}\left(g_{*} \cdot \xi_{x}\right)
$$

is a consequence of equation (A2).

The problem of determining which bundles allow the dependence on the fibre coordinate to be eliminated has been considered previously [76][17]. A complete proof of the restriction to bundles with fibre $G / D$, where $G$ is a Lie group and $D$ a discrete subgroup, is given in the following theorem.

A necessary condition is

$$
T \psi_{\beta \alpha}^{x} \phi_{y} \Gamma^{\alpha}\left(\xi_{x}\right)=\phi_{y^{\prime}} A(x) \Gamma^{\alpha}\left(\xi_{x}\right)
$$


or equivalently, that there exists a global diffeomorphism which is a solution to the differential system

$$
T \psi_{\beta \alpha}^{x}=\phi_{y^{\prime}} \cdot A \cdot \phi_{y}^{-1}
$$

By Frobenius' theorem, the integrability conditions for the differential system require that the commutator of vector fields on the fibre are invariant with respect to the map $\phi_{y^{\prime}} \cdot A \cdot \phi_{y}^{-1}$. Thus,

$$
\left[\phi_{y^{\prime}} \cdot A \cdot \phi_{y}^{-1} X, \phi_{y^{\prime}} \cdot A \cdot \phi_{y}^{-1} Y\right]_{y_{0}^{\prime}}=\phi_{y^{\prime}} \cdot A \phi_{y}^{-1}[X, Y]_{y_{0}}
$$

for any vector fields $X, Y \in T F$, implying

$$
c_{i j k}\left(y_{0}\right) A_{m k}=c_{k l m}\left(y_{0}^{\prime}\right) A_{k i} A_{l j}
$$

with the coefficients $c_{i j k}(y)$ given by $\left[\xi_{i}(y), \xi_{j}(y)\right]=c_{i j k}(y) \xi_{k}(y)$, with $\xi_{i}(y)=\phi_{y} \cdot e_{i}, e_{i} \in V$ representing an orthonormal basis for $T_{y}(F)$.

The most general gauge matrix A that can be allowed is $A=A_{1}\left(y^{\prime}\right) A_{2}(x) A_{1}(y)^{-1}$ as this gives

$$
T \psi_{\beta \alpha}^{x}=\phi_{y^{\prime}} \cdot A_{1}\left(y^{\prime}\right) A_{2}(x) A_{1}(y)^{-1} \cdot \phi_{y}^{-1}=\phi_{y^{\prime}}^{\prime} \cdot A_{2}(x) \cdot \phi_{y}^{\prime-1}
$$

if the new parallelism $\phi_{y}^{\prime}$ is given by $\phi_{y} \cdot A_{1}(y)$. The integrability condition for the differential system given by equation (A8) is

$$
\begin{aligned}
{\left[\phi_{y^{\prime}} \cdot A_{1}\left(y^{\prime}\right) A_{2}(x) A_{1}(y)^{-1} \cdot \phi_{y}^{-1} \cdot X\right.} & \left., \phi_{y^{\prime}} \cdot A_{1}\left(y^{\prime}\right) A_{2}(x) A_{1}(y)^{-1} \cdot \phi_{y}^{-1} \cdot Y\right]_{y_{0}^{\prime}} \\
& =\phi_{y_{0}^{\prime}} \cdot A_{1}\left(y^{\prime}\right) A_{2}(x) A_{1}(y)^{-1} \cdot \phi_{y_{0}}^{-1} \cdot[X, Y]_{y_{0}}
\end{aligned}
$$

Letting $X=\xi_{i}(y)=\phi_{y} \cdot e_{i}$, one finds that

$$
c_{i j k}\left[A_{1}\left(y_{0}^{\prime}\right) \cdot A_{2}(x) \cdot A_{1}\left(y_{0}\right)^{-1}\right]_{m k}=c_{k l m}\left[A_{1}\left(y_{0}^{\prime}\right) A_{2}(x) A_{1}\left(y_{0}\right)^{-1}\right]_{k i}\left[A_{1}\left(y_{0}^{\prime}\right) A_{2}(x) \cdot A_{1}\left(y_{0}\right)^{-1}\right]_{l j}
$$

Defining the basis $\xi_{i}^{\prime}(y)$ using the new parallelism $\phi_{y}^{\prime} \cdot e_{i}=\phi_{y} \cdot A_{1}(y) e_{i}$, and the structure coefficients $c_{i j k}^{\prime}(y)$ by the commutation relations $\left[\xi_{i}^{\prime}, \xi_{j}^{\prime}\right]_{y}=c_{i j k}^{\prime}(y) \xi_{k}^{\prime}(y)$, it follows that

$$
c_{i j k}^{\prime}(y)\left(A_{1}(y)\right)_{n k}=c_{l m n}\left(A_{1}(y)\right)_{l i}\left(A_{1}(y)\right)_{m j}
$$

Using this relation and multiplying equation (A11) by $\left(A_{1}\left(y_{0}\right)\right)_{i r}\left(A_{1}\left(y_{0}\right)\right)_{j s}\left(A^{-1}\left(y_{0}^{\prime}\right)\right)_{p m}$ gives

$$
c_{r s n}^{\prime}\left(y_{0}\right) A_{2 p n}=c_{l m p}^{\prime}\left(y_{0}^{\prime}\right) A_{2 l r} A_{2 m s}
$$

which is equivalent to the previous condition (A8).

It may be noted that the proof of the existence of a globally integrable parallelism on the standard fibre depends on the following lemma, with a proof that provides further details of the demonstration given in reference [17]:

Lemma. The set $\mathcal{C}_{y_{1}}=\left\{y \in F \mid c_{i j k}(y)=c_{i j k}\left(y_{1}\right)\right\}$ either contains all of $\mathrm{F}$ or is a set of dimensionality less than one in $\mathrm{F}$.

Proof. An identity satisfied by the vector fields $\left\{\xi_{i}\right\}$ induced by the parallelism $\phi_{y}$ [77] implies that

$$
c_{i j l}\left(y^{\prime}\right) c_{k l m}\left(y^{\prime}\right)-\xi_{k}\left(c_{i j m}\right)\left(y^{\prime}\right)=c_{i j l}(y) c_{k l m}(y)-\xi_{k}\left(c_{i j m}\right)(y)
$$


where $\xi_{k}\left(c_{i j m}\right)$ is defined by the action of the vector field on a function on $\mathrm{F}$ [17]. Assuming initially that $\mathcal{C}_{y_{1}}$ is a continuous curve through $y_{1}$ with tangent vector $X_{y_{1}}=X_{k} \xi_{k}\left(y_{1}\right)$ and contracting equation (A16) by $X_{k}$,

$$
c_{i j l}(y) c_{k l m}(y) X_{k}-X_{k} \xi_{k}\left(c_{i j m}\right)(y)=c_{i j l}\left(y_{1}\right) c_{k l m}\left(y_{1}\right) X_{k}-X_{k} \xi_{k}\left(c_{i j m}\right)\left(y_{1}\right)
$$

which implies, on $\mathcal{C}_{y_{1}}$, that $X_{k} \xi_{k}\left(c_{i j m}\right)(y)=X_{k} \xi_{k}\left(c_{i j m}\right)\left(y_{1}\right)=0$ when $X_{k}$ is constant. Similarly, the vanishing of the derivative of $c_{i j l}(y) c_{k l m}(y)-\xi_{k}\left(c_{i j m}\right)(y)$ by equation (A16) gives

$$
\begin{array}{r}
\left(X_{n} \xi_{n}\left(c_{i j l}\right)\left(y_{1}\right)\right) X_{k} c_{k l m}\left(y_{1}\right)+X_{k} c_{i j l}\left(y_{1}\right)\left(X_{n} \xi_{n}\left(c_{k l m}\right)\left(y_{1}\right)\right)-X_{n} \xi_{n} X_{k} \xi_{k}\left(c_{i j m}\right)\left(y_{1}\right) \\
=-X_{n} \xi_{n} X_{k} \xi_{k}\left(c_{i j m}\right)\left(y_{1}\right)=0
\end{array}
$$

and repeated differentiation leads to $X_{n_{1}} \xi_{n_{1}} \ldots X_{n_{r}} \xi_{n_{r}}\left(c_{i j m}\right)\left(y_{1}\right)=0$. Consequently, $c_{i j m}$ is constant along the integral curve of $\mathrm{X}$, and using the assumption that the set $\mathcal{C}_{y_{1}}$ is at least one-dimensional, one may conclude that it contains the integral curve of a parallel vector field $\mathrm{X}$ passing through $y_{1}$.

A two-dimensional surface $S_{Y}$ is spanned by the integral curves of another parallel vector field Y intersecting $\mathcal{C}_{y_{1}}$ and an n-dimensional neighbourhood of $y_{1}$ is covered by the surfaces $S_{\sum_{i} a_{i} Y_{i}}$ corresponding to arbitrary linear combinations of the independent vector fields $Y_{1}, \ldots, Y_{n-1}$. The surfaces $S_{Y}$ and $S_{Y^{\prime}}$ will initially coincide at $\mathcal{C}_{y_{1}}$ if $Y^{\prime}=a X+b Y$. However, translation of the vector field $\mathrm{X}$ a distance $\mathrm{t}$ along the integral curve of $\mathrm{Y}$ using the one-parameter family of diffeomorphisms $\left\{\chi_{t}\right\}$ gives a vector field

$$
\chi_{t *} X=X-t[X, Y]+\frac{t^{2}}{2}[[X, Y], Y]-\ldots
$$

Parallel transport of the vector field $\mathrm{X}$ along the integral curve of $Y^{\prime}$ using the one-parameter family of diffeomorphisms $\left\{\chi_{v *}^{\prime}\right\}$ gives the vector field

$$
\chi_{v *}^{\prime} X=X-b v[X, Y]+b^{2} \frac{v^{2}}{2}[[X, Y], Y]-\ldots+a b \frac{v^{2}}{2}[[X, Y], X]-\ldots
$$

Even after setting t equal to $\sum_{j} b_{j} v^{j}$, with $b_{1}=b$, higher-order terms such as that containing $a b \frac{v^{2}}{2}$ will vitiate the possibility of equating $\chi_{t *} X$ with $\chi_{v *}^{\prime} X$. Thus, the tangent spaces to $S_{Y}$ and $S_{Y^{\prime}}$, spanned by the bases $\left\{\chi_{t *} X, Y\right\}$ and $\left\{\chi_{v *}^{\prime} X, Y^{\prime}\right\}\left\{\chi_{v *}^{\prime} X, a X+b Y\right\}$ respectively, are equivalent at $\mathcal{C}_{y_{1}}$ but differ when $t, v \neq 0$. Since $S_{Y^{\prime}}$ does not always coincide with $S_{Y}$, it must intersect with a surface $S_{Y^{\prime \prime}}$, where $Y^{\prime \prime}$ is another linear combination of the vector fields $Y_{1}, \ldots, Y_{n-1}, \sum_{i} a_{i}^{\prime \prime} Y_{i}$.

Translation of $\mathcal{C}_{y_{1}}$ a distance $v$ along integral curves of $Y^{\prime}$ results in a curve of constant $c_{i j k}$, $\mathcal{C}_{\chi_{v}^{\prime}\left(y_{1}\right)}$, because

$$
\left(Y_{n}^{\prime} \xi_{n} c_{i j l}(y)\right) Y_{k}^{\prime} c_{k l m}(y)+\left(Y_{k}^{\prime} c_{i j l}(y)\right)\left(Y_{n}^{\prime} \xi_{n} c_{k l m}(y)\right)-Y_{n}^{\prime} \xi_{n} Y_{k}^{\prime} \xi_{k}\left(c_{i j m}\right)(y)=0
$$

together with equation (A19) implies that

$$
Y_{n}^{\prime} \xi_{n} Y_{k}^{\prime} \xi_{k}\left(c_{i j m}\right)(y)=Y_{n}^{\prime} \xi_{n} Y_{k}^{\prime} \xi_{k}\left(c_{i j m}\right)\left(\chi_{v}^{\prime}\left(y_{1}\right)\right)
$$

and repeated differentiation gives

$$
Y_{n_{1}}^{\prime} \xi_{n_{1}} \ldots Y_{n_{r}}^{\prime} \xi_{n_{r}}\left(c_{i j m}\right)(y)=Y_{n_{1}}^{\prime} \xi_{n_{1}} \ldots Y_{n_{r}}^{\prime} \xi_{n_{r}}\left(c_{i j m}\right)\left(\chi_{v}^{\prime}\left(y_{1}\right)\right) \quad \forall y \in \mathcal{C}_{y_{1}}
$$


Similarly, translation of $\mathcal{C}_{y_{1}}$ a distance w along the integral curves of $Y^{\prime \prime}$ produces another curve of constant $c_{i j k}, \mathcal{C}_{\chi_{w}^{\prime \prime}\left(y_{1}\right)}$.

If a point $y_{3}$ lies in the intersection of $S_{Y^{\prime}}$ and $S_{Y^{\prime \prime}}$, then it must be located on an a curve $\mathcal{C}_{\chi_{v}^{\prime}\left(y_{1}\right)}$ for some $\mathrm{v}$ and $\mathcal{C}_{\chi_{w}^{\prime \prime}\left(y_{1}\right)}$ for some w. Parallel transport of one of these curves along the other will sweep out a two-dimensional surface of constant $c_{i j k}$. When this surface is translated back to $y_{1}$, one obtains a two-dimensional neighbourhood of constant $c_{i j k}$ about the point $y_{1}$. This procedure can be repeated until an n-dimensional neighbourhood of $y_{1}$ of constant $c_{i j k}$ is constructed. If $\mathrm{F}$ is compact, it may be covered by a finite number of these neighbourhoods and $\mathcal{C}_{y_{1}}$ contains all of F. Otherwise, it has dimensionality less than one.

This lemma has been used previously [17] to demonstrate that the standard fibre is $G / D$, where $\mathrm{G}$ is a Lie group and $\mathrm{D}$ is a discrete subgroup. When the standard fibre is a group, and the gauge matrix A represents the adjoint action of $g^{-1}$, then if $g=\exp \left(t_{j} X_{j}\right)$,

$$
\begin{aligned}
\exp \left(-t_{j} X_{j}\right) X_{i} \exp \left(t_{j} X_{j}\right) & =X_{m}\left(\delta_{i m}-t_{j} c_{i j m}+\frac{1}{2} t_{j} t_{k} c_{i k l} c_{l j m}+\ldots\right) \\
A_{m i} & =\delta_{m i}-t_{j} c_{i j m}+\frac{1}{2} t_{j} t_{k} c_{i k l} c_{l j m}+\ldots
\end{aligned}
$$

Substituting the formula for $A_{m i}$ into equation (A8) and equating the coefficients at each order in $\mathrm{t}$ gives

$$
\begin{aligned}
O\left(t^{0}\right): c_{i j m} & =c_{i j m} \\
O(t): c_{i j k} c_{k l m} & =c_{i l k} c_{k j m}+c_{i k m} c_{k j l} \\
O\left(t^{2}\right): \quad c_{i j k} c_{k l p} c_{l n m} & =c_{k j m} c_{i p t} c_{t n k}+c_{i l m} c_{j p t} c_{t n l}-2 c_{k l m} c_{i n k} c_{j p l}
\end{aligned}
$$

The relations for $O\left(t^{r}\right), r \geq 1$ all follow from the Jacobi identity for the Lie algebra structure constants.

One may wish to consider a more general linear transformation A of the form

$$
A_{m i}=\delta_{m i}-t_{j} d_{1 i j m}+\frac{1}{2} t_{j} t_{k} d_{2 i k l} d_{3 l j m}+\ldots
$$

leading to the relations

$$
\begin{aligned}
c_{i j k} d_{1 k l m} & =d_{1 i l k} c_{k j m}+c_{i k m} d_{1 k j l} \\
c_{i j k} d_{2 k l p} d_{3 l n m} & =c_{k j m} d_{2 i p t} d_{3 t n k}+c_{i l m} d_{2 j p t} d_{3 t n l}-2 c_{k l m} d_{1 i n k} d_{1 j p l}
\end{aligned}
$$

Equation (A27) comprises of $n^{2}$ relations for the $n^{3}$ components of the tensor $d_{1 i j k}$, so that it does not fully determine $d_{1}$. Similarly, equation (A28) consists of $n^{5}$ relations for the $3 n^{3}$ components of $d_{1 i j k}, d_{2 i j k}$ and $d_{3 i j k}$. Higher orders of $t$ give rise to equations which will include a larger number of conditions on the coefficients $d_{M i j k}$. Even if some of the constraints are redundant, the arbitrariness in the choice of $d_{M i j k}$ can be eliminated, leaving coefficients that are equal to the structure constants $c_{i j k}$. A similar conclusion about the effect of the independence of Lagrangian with respect to the fibre coordinates on the category or bundles has been obtained in a different proof [76]. 


\section{B. Gauge Transformation Constraints for $\mathrm{SO}(8)$ and $\mathrm{SU}(4)$ Actions on an $S^{7}$ Bundle}

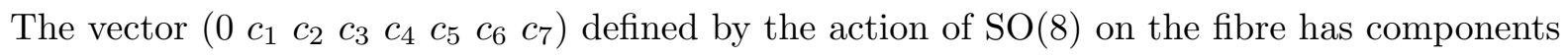

$$
\begin{aligned}
& c_{1}=d_{01}\left(y_{0}^{2}+y_{1}^{2}\right)+\left(d_{02}-d_{13}\right)\left(y_{1} y_{2}+y_{0} y_{3}\right)+\left(d_{03}+d_{12}\right)\left(y_{1} y_{3}-y_{0} y_{2}\right) \\
& +\left(d_{04}-d_{15}\right)\left(y_{1} y_{4}+y_{0} y_{5}\right)+\left(d_{05}+d_{14}\right)\left(y_{1} y_{5}-y_{0} y_{4}\right)+\left(d_{06}-d_{17}\right)\left(y_{1} y_{6}+y_{0} y_{7}\right) \\
& +\left(d_{07}+d_{16}\right)\left(y_{1} y_{7}-y_{0} y_{6}\right)-d_{23}\left(y_{2}^{2}+y_{3}^{2}\right)+\left(-d_{24}-d_{35}\right)\left(y_{3} y_{4}-y_{2} y_{5}\right) \\
& +\left(-d_{25}+d_{34}\right)\left(y_{3} y_{5}+y_{2} y_{4}\right)+\left(-d_{26}-d_{37}\right)\left(y_{3} y_{6}-y_{2} y_{7}\right)+\left(-d_{27}+d_{36}\right)\left(y_{3} y_{7}+y_{2} y_{6}\right) \\
& -d_{45}\left(y_{4}^{2}+y_{5}^{2}\right)+\left(-d_{46}-d_{57}\right)\left(y_{5} y_{6}-y_{4} y_{7}\right)+\left(-d_{47}+d_{56}\right)\left(y_{5} y_{7}+y_{4} y_{6}\right) \\
& -d_{67}\left(y_{6}^{2}+y_{7}^{2}\right) \\
& c_{2}=\left(d_{01}+d_{23}\right)\left(y_{1} y_{2}-y_{0} y_{3}\right)+d_{02}\left(y_{0}^{2}+y_{2}^{2}\right)+\left(d_{03}+d_{12}\right)\left(y_{2} y_{3}+y_{0} y_{1}\right) \\
& +\left(d_{04}-d_{26}\right)\left(y_{2} y_{4}+y_{0} y_{6}\right)+\left(d_{05}+d_{27}\right)\left(y_{2} y_{5}-y_{0} y_{7}\right)+\left(d_{06}+d_{24}\right)\left(y_{2} y_{6}-y_{0} y_{4}\right) \\
& +\left(d_{07}-d_{25}\right)\left(y_{2} y_{7}+y_{0} y_{5}\right)+d_{13}\left(y_{1}^{2}+y_{3}^{2}\right)+\left(d_{14}-d_{36}\right)\left(y_{3} y_{4}+y_{1} y_{6}\right) \\
& +\left(d_{15}+d_{37}\right)\left(y_{3} y_{5}-y_{1} y_{7}\right)+\left(d_{16}+d_{34}\right)\left(y_{3} y_{6}-y_{1} y_{4}\right)+\left(d_{17}-d_{35}\right)\left(y_{3} y_{7}+y_{1} y_{5}\right) \\
& +\left(d_{45}-d_{67}\right)\left(-y_{4} y_{7}-y_{5} y_{6}\right)-d_{46}\left(y_{4}^{2}+y_{6}^{2}\right)+\left(d_{47}-d_{56}\right)\left(-y_{6} y_{7}+y_{4} y_{5}\right) \\
& +d_{57}\left(y_{5}^{2}+y_{7}^{2}\right) \\
& c_{3}=\left(d_{01}+d_{23}\right)\left(y_{1} y_{3}+y_{0} y_{2}\right)+\left(d_{02}-d_{13}\right)\left(y_{2} y_{3}-y_{0} y_{1}\right)+d_{03}\left(y_{0}^{2}+y_{3}^{2}\right) \\
& +\left(d_{04}+d_{37}\right)\left(y_{3} y_{4}-y_{0} y_{7}\right)+\left(d_{05}+d_{36}\right)\left(y_{3} y_{5}-y_{0} y_{6}\right)+\left(d_{06}-d_{35}\right)\left(y_{3} y_{6}+y_{0} y_{5}\right) \\
& +\left(d_{07}-d_{34}\right)\left(y_{3} y_{7}+y_{0} y_{4}\right)-d_{12}\left(y_{1}^{2}+y_{2}^{2}\right)+\left(d_{14}-d_{27}\right)\left(-y_{2} y_{4}-y_{1} y_{7}\right) \\
& +\left(d_{15}-d_{26}\right)\left(-y_{2} y_{5}-y_{1} y_{6}\right)+\left(d_{16}+d_{25}\right)\left(-y_{2} y_{6}+y_{1} y_{5}\right)+\left(d_{17}+d_{24}\right)\left(-y_{2} y_{7}+y_{1} y_{4}\right) \\
& +\left(d_{45}-d_{67}\right)\left(y_{5} y_{7}-y_{4} y_{6}\right)+\left(d_{46}+d_{57}\right)\left(y_{6} y_{7}+y_{4} y_{5}\right)+d_{47}\left(y_{4}^{2}+y_{7}^{2}\right) \\
& +d_{56}\left(y_{5}^{2}+y_{6}^{2}\right) \\
& c_{4}=\left(d_{01}+d_{45}\right)\left(y_{1} y_{4}-y_{0} y_{5}\right)+\left(d_{02}+d_{46}\right)\left(y_{2} y_{4}-y_{0} y_{6}\right)+\left(d_{03}-d_{47}\right)\left(y_{3} y_{4}+y_{0} y_{7}\right) \\
& +d_{04}\left(y_{0}^{2}+y_{4}^{2}\right)+\left(d_{05}+d_{14}\right)\left(y_{4} y_{5}+y_{0} y_{1}\right)+\left(d_{06}+d_{24}\right)\left(y_{4} y_{6}+y_{0} y_{2}\right) \\
& +\left(d_{07}-d_{34}\right)\left(y_{4} y_{7}-y_{0} y_{3}\right)+\left(d_{12}+d_{56}\right)\left(y_{2} y_{5}-y_{1} y_{6}\right)+\left(d_{13}-d_{57}\right)\left(y_{3} y_{5}+y_{1} y_{7}\right) \\
& +d_{15}\left(y_{1}^{2}+y_{5}^{2}\right)+\left(d_{16}+d_{25}\right)\left(y_{5} y_{6}+y_{1} y_{2}\right)+\left(d_{17}-d_{35}\right)\left(d_{5} d_{7}-y_{1} y_{3}\right) \\
& +\left(d_{23}-d_{67}\right)\left(y_{3} y_{6}+y_{2} y_{7}\right)+d_{26}\left(y_{2}^{2}+y_{6}^{2}\right)+\left(d_{27}-d_{36}\right)\left(y_{6} y_{7}-y_{2} y_{3}\right) \\
& -d_{37}\left(y_{3}^{2}+y_{7}^{2}\right) \\
& c_{5}=\left(d_{01}+d_{45}\right)\left(y_{1} y_{5}+y_{0} y_{4}\right)+\left(d_{02}-d_{57}\right)\left(y_{2} y_{5}+y_{0} y_{7}\right)+\left(d_{03}-d_{56}\right)\left(y_{3} y_{5}+y_{0} y_{6}\right) \\
& +\left(d_{04}-d_{15}\right)\left(y_{4} y_{5}-y_{0} y_{1}\right)+d_{05}\left(y_{0}^{2}+y_{5}^{2}\right)+\left(d_{06}-d_{35}\right)\left(y_{5} y_{6}-y_{0} y_{3}\right) \\
& +\left(d_{07}-d_{25}\right)\left(y_{5} y_{7}-y_{0} y_{2}\right)+\left(d_{12}+d_{47}\right)\left(-y_{2} y_{4}+y_{1} y_{7}\right)+\left(d_{13}+d_{46}\right)\left(-y_{3} y_{4}+y_{1} y_{6}\right) \\
& -d_{14}\left(y_{1}^{2}+y_{4}^{2}\right)+\left(d_{16}+d_{34}\right)\left(-y_{4} y_{6}-y_{1} y_{3}\right)+\left(d_{17}+d_{24}\right)\left(-y_{4} y_{7}-y_{1} y_{2}\right) \\
& +\left(d_{23}-d_{67}\right)\left(-y_{3} y_{7}+y_{2} y_{6}\right)+\left(d_{26}+d_{37}\right)\left(-y_{6} y_{7}-y_{2} y_{3}\right)-d_{27}\left(y_{2}^{2}+y_{7}^{2}\right) \\
& -d_{36}\left(y_{3}^{2}+y_{6}^{2}\right) \\
& c_{6}=\left(d_{01}+d_{67}\right)\left(y_{1} y_{6}-y_{0} y_{7}\right)+\left(d_{02}+d_{46}\right)\left(y_{2} y_{6}+y_{0} y_{4}\right)+\left(d_{03}-d_{56}\right)\left(y_{3} y_{6}-y_{0} y_{5}\right) \\
& +\left(d_{04}-d_{26}\right)\left(y_{4} y_{6}-y_{0} y_{2}\right)+\left(d_{05}+d_{36}\right)\left(y_{5} y_{6}+y_{0} y_{3}\right)+d_{06}\left(y_{0}^{2}+y_{6}^{2}\right) \\
& +\left(d_{07}+d_{16}\right)\left(y_{6} y_{7}+y_{0} y_{1}\right)+\left(d_{12}+d_{47}\right)\left(y_{2} y_{7}+y_{1} y_{4}\right)+\left(d_{13}-d_{57}\right)\left(y_{3} y_{7}-y_{1} y_{5}\right)
\end{aligned}
$$




$$
\begin{aligned}
& +\left(d_{14}-d_{27}\right)\left(y_{4} y_{7}-y_{1} y_{2}\right)+\left(d_{15}+d_{37}\right)\left(y_{5} y_{7}+y_{1} y_{3}\right)+d_{17}\left(y_{1}^{2}+y_{7}^{2}\right) \\
& +\left(d_{23}-d_{45}\right)\left(-y_{3} y_{4}-y_{2} y_{5}\right)-d_{24}\left(y_{2}^{2}+y_{4}^{2}\right)+\left(d_{25}-d_{34}\right)\left(-y_{4} y_{5}+y_{2} y_{3}\right) \\
& +d_{35}\left(y_{3}^{2}+y_{5}^{2}\right) \\
c_{7} & =\left(d_{01}+d_{67}\right)\left(y_{1} y_{7}+y_{0} y_{6}\right)+\left(d_{02}-d_{57}\right)\left(y_{2} y_{7}-y_{0} y_{5}\right)+\left(d_{03}-d_{47}\right)\left(y_{3} y_{7}-y_{0} y_{4}\right) \\
& +\left(d_{04}+d_{37}\right)\left(y_{4} y_{7}+y_{0} y_{3}\right)+\left(d_{05}+d_{27}\right)\left(y_{5} y_{7}+y_{0} y_{2}\right)+\left(d_{06}-d_{17}\right)\left(y_{6} y_{7}-y_{0} y_{1}\right) \\
& +d_{07}\left(y_{0}^{2}+y_{7}^{2}\right)+\left(d_{12}+d_{56}\right)\left(-y_{2} y_{6}-y_{1} y_{5}\right)+\left(d_{13}+d_{46}\right)\left(-y_{3} y_{6}-y_{1} y_{4}\right) \\
& +\left(d_{14}-d_{36}\right)\left(-y_{4} y_{6}+y_{1} y_{3}\right)+\left(d_{15}-d_{26}\right)\left(-y_{5} y_{6}+y_{1} y_{2}\right)-d_{16}\left(y_{1}^{2}+y_{6}^{2}\right) \\
& +\left(d_{23}-d_{45}\right)\left(-y_{2} y_{4}+y_{3} y_{5}\right)+\left(d_{24}+d_{35}\right)\left(y_{4} y_{5}+y_{2} y_{3}\right)+d_{25}\left(y_{2}^{2}+y_{5}^{2}\right) \\
& +d_{34}\left(y_{3}^{2}+y_{4}^{2}\right)
\end{aligned}
$$

The general SU(4) matrix is given by $g=D\left(\delta_{1}, \delta_{2}, \delta_{3},-\delta_{1}-\delta_{2}-\delta_{3}\right) \cdot U_{23}\left(\phi_{3}, \sigma_{6}\right)$ $U_{12}\left(\theta_{3}, \sigma_{5}\right) U_{13}\left(\phi_{2}, \sigma_{4}\right) \cdot U_{01}\left(\theta_{2}, \sigma_{3}\right) U_{02}\left(\theta_{1}, \sigma_{2}\right) U_{03}\left(\phi_{1}, \sigma_{1}\right)$, where $D\left(\delta_{1}, \delta_{2}, \delta_{3},-\delta_{1}-\delta_{2}-\delta_{3}\right)$ is the diagonal matrix with elements $e^{i \delta_{1}}, e^{i \delta_{2}}, e^{i \delta_{3}}, e^{-i \delta_{1}-\delta_{2}-\delta_{3}}$ and $U_{p q}(\phi, \sigma)$, which has all diagonal elements equal to 1 , except for $u_{p p}$ and $u_{q q}$, which should be $\cos \phi$, and non-zero off-diagonal entries $u_{p q}=-\sin \phi e^{-i \sigma}, u_{q p}=\sin \phi e^{i \sigma}$,

represents a unitary transformation in the (p,q)-plane. Consequently, the elements of $g$ are

$$
\begin{aligned}
g_{00}= & \cos \theta_{2} \cos \theta_{1} \cos \phi_{1} e^{i \delta_{1}} \\
g_{01}= & -\sin \theta_{2} e^{i\left(\delta_{1}-\sigma_{3}\right)} \\
g_{02}= & -\cos \theta_{2} \sin \theta_{1} e^{\left(\delta_{1}-\sigma_{2}\right)} \\
g_{03}= & -\cos \theta_{2} \cos \theta_{1} \sin \phi_{1} e^{i\left(\delta_{1}-\sigma_{1}\right)} \\
g_{10}= & \cos \theta_{3} \sin \theta_{2} \cos \theta_{1} \cos \phi_{1} e^{i\left(\delta_{2}+\sigma_{3}\right)} \\
& \quad-\sin \theta_{3} \sin \theta_{1} \cos \phi_{1} e^{i\left(\sigma_{2}+\delta_{2}-\sigma_{5}\right)}-\cos \theta_{3} \sin \phi_{2} \sin \phi_{1} e^{i\left(\sigma_{1}+\delta_{2}-\sigma_{4}\right)} \\
g_{11}= & \cos \theta_{2} \cos \theta_{3} \cos \phi_{2} e^{i \delta_{2}} \\
g_{12}= & -\cos \theta_{3} \cos \phi_{2} \sin \theta_{2} \sin \theta_{1} e^{i\left(\delta_{2}+\sigma_{3}-\sigma_{2}\right)}-\sin \theta_{3} \cos \theta_{1} e^{i\left(\delta_{2}-\sigma_{5}\right)} \\
g_{13}= & -\cos \theta_{3} \cos \phi_{2} \sin \theta_{2} \sin \phi_{1} \cos \theta_{1} e^{i\left(\delta_{2}+\sigma_{3}-\sigma_{1}\right)} \\
& \quad+\sin \theta_{3} \sin \theta_{1} \sin \phi_{1} e^{i\left(\delta_{2}+\sigma_{2}-\sigma_{1}-\sigma_{5}\right)} \\
& \quad-\cos \theta_{3} \sin \phi_{2} \cos \phi_{1} e^{i\left(\delta_{2}-\sigma_{4}\right)}
\end{aligned}
$$


$g_{20}=\cos \phi_{3} \sin \theta_{3} \cos \phi_{2} \sin \theta_{2} \cos \theta_{1} \cos \phi_{1} e^{i\left(\delta_{3}+\sigma_{3}+\sigma_{5}\right)}$

$+\cos \theta_{3} \cos \phi_{3} \sin \theta_{1} \cos \phi_{1} e^{i\left(\delta_{3}+\sigma_{2}\right)}$

$-\cos \phi_{3} \sin \theta_{3} \sin \phi_{2} \sin \phi_{1} e^{i\left(\sigma_{1}+\delta_{3}+\sigma_{5}-\sigma_{4}\right)}$

$g_{21}=\cos \phi_{3} \sin \theta_{3} \cos \phi_{2} \cos \theta_{2} e^{i\left(\delta_{3}+\sigma_{5}\right)}$

$g_{22}=-\cos \phi_{3} \sin \theta_{3} \cos \phi_{2} \sin \theta_{2} \sin \theta_{1} e^{i\left(\delta_{3}+\sigma_{3}+\sigma_{5}-\sigma_{2}\right)}$

$+\cos \phi_{3} \cos \theta_{3} \cos \theta_{1} e^{i \delta_{3}}$

$g_{23}=-\cos \phi_{3} \sin \theta_{3} \cos \phi_{2} \sin \theta_{2} \cos \theta_{1} \sin \phi_{1} e^{i\left(\delta_{3}+\sigma_{3}-\sigma_{1}\right)}$

$-\cos \phi_{3} \cos \theta_{3} \sin \theta_{1} \sin \phi_{1} e^{i\left(\delta_{3}+\sigma_{2}-\sigma_{1}\right)}$

$-\cos \phi_{3} \sin \theta_{3} \sin \phi_{2} \cos \phi_{1} e^{i\left(\delta_{3}+\sigma_{5}-\sigma_{4}\right)}$

$g_{30}=\sin \phi_{3} \sin \theta_{3} \cos \phi_{2} \sin \theta_{2} \cos \theta_{1} \cos \phi_{1} e^{i\left(\sigma_{3}+\sigma_{5}+\sigma_{6}-\delta_{1}-\delta_{2}-\delta_{3}\right)}$

$+\cos \phi_{3} \sin \phi_{2} \sin \theta_{2} \cos \theta_{1} \cos \phi_{1} e^{i\left(\sigma_{3}+\sigma_{4}-\delta_{1}-\delta_{2}-\delta_{3}\right)}$

$+\sin \phi_{3} \cos \theta_{3} \sin \theta_{1} \cos \phi_{1} e^{i\left(\sigma_{2}+\sigma_{6}-\delta_{1}-\delta_{2}-\delta_{3}\right)}$

$+\cos \phi_{3} \cos \phi_{2} \sin \phi_{1} e^{i\left(\sigma_{1}-\delta_{1}-\delta_{2}-\delta_{3}\right)}$

$-\sin \phi_{3} \sin \theta_{3} \sin \theta_{2} \sin \phi_{1} e^{i\left(\sigma_{1}+\sigma_{5}+\sigma_{6}-\sigma_{4}-\delta_{1}-\delta_{2}-\delta_{3}\right)}$

$g_{31}=\sin \phi_{3} \sin \theta_{3} \cos \phi_{2} \cos \theta_{2} e^{i\left(\sigma_{5}+\sigma_{6}-\delta_{1}-\delta_{2}-\delta_{3}\right)}$

$+\cos \phi_{3} \sin \phi_{2} \cos \theta_{2} e^{i\left(\sigma_{4}-\delta_{1}-\delta_{2}-\delta_{3}\right)}$

$g_{32}=-\sin \phi_{3} \sin \theta_{3} \cos \phi_{2} \sin \theta_{2} \sin \theta_{1} e^{i\left(\sigma_{3}+\sigma_{5}-\sigma_{2}-\sigma_{6}-\delta_{1}-\delta_{2}-\delta_{3}\right)}$

$-\cos \phi_{3} \sin \theta_{2} \sin \phi_{2} e^{i\left(\sigma_{3}+\sigma_{4}-\sigma_{2}-\delta_{1}-\delta_{2}-\delta_{3}\right)}$

$-\sin \phi_{3} \cos \theta_{3} \cos \theta_{1} e^{i\left(\sigma_{6}-\delta_{1}-\delta_{2}-\delta_{3}\right)}$

$g_{33}=-\sin \phi_{3} \cos \theta_{3} \sin \theta_{1} \sin \phi_{1} e^{i\left(\sigma_{2}+\sigma_{6}-\sigma_{1}-\delta_{1}-\delta_{2}-\delta_{3}\right)}$

$-\sin \phi_{3} \sin \theta_{3} \cos \phi_{2} \sin \theta_{2} \cos \theta_{1} \sin \phi_{1} e^{\left(\sigma_{3}+\sigma_{5}+\sigma_{6}-\sigma_{1}-\delta_{1}-\delta_{2}-\delta_{3}\right)}$

$-\cos \phi_{3} \sin \phi_{2} \sin \theta_{2} \cos \theta_{1} \cos \phi_{1} e^{i\left(\sigma_{3}+\sigma_{4}-\sigma_{1}-\delta_{1}-\delta_{2}-\delta_{3}\right)}$

$-\sin \phi_{3} \sin \theta_{3} \sin \phi_{2} \cos \phi_{1} e^{i\left(\sigma_{5}+\sigma_{6}-\sigma_{4}-\delta_{1}-\delta_{2}-\delta_{3}\right)}$

$+\cos \phi_{3} \cos \phi_{2} \cos \phi_{1} e^{-i\left(\delta_{1}+\delta_{2}+\delta_{3}\right)}$

\section{An Application to Quantum Principal Bundles}

The concepts of principal bundles and gauge transformations can be generalized using quantum groups. The bundle $P=P(B, A)$ is a quantum principal bundle [78] with quantum structure group $\mathrm{A}$ and base $\mathrm{B}$ if

(i) A is a Hopf algebra with co-product $\Delta: A \rightarrow A \otimes A$, co-unit $\epsilon: A \rightarrow k$, and antipode map $S: A \rightarrow A$.

(ii) $\left(P, \Delta_{R}: P \rightarrow P \otimes A\right)$ is a right A-co-module algebra

(iii) $B=P^{A}=\left\{u \in P: \Delta_{R} u=u \otimes 1\right\}$

(iv) $(\cdot \otimes i d)\left(i d \otimes \Delta_{R}\right): P \otimes P \rightarrow P \otimes A$ is a surjection

(v) ker $\sim \Gamma_{\text {hor }}$ where $\Gamma_{\text {hor }}=\operatorname{Pj}\left(\Gamma_{B}\right) P \subset \Gamma_{P}$, with $j: \Omega B \hookrightarrow \Omega P$ being an inclusion and $\Gamma_{B}$ being the space of one-forms on $\mathrm{B}$, and $\sim=\left.(\cdot \otimes i d) \circ\left(i d \otimes \Delta_{R}\right)\right|_{P^{2}}: \Gamma_{P} \rightarrow P \otimes A$. 
Although the Hopf algebra acts on the co-module algebra $\mathrm{P}$ in the principal quantum bundle, the map $(\cdot \otimes i d) \circ\left(i d \otimes \Delta_{R}\right): P \otimes P \rightarrow P \otimes A$ descends to an isomorphism $P \otimes_{B} P \rightarrow P \otimes A$. By analogy with classical bundles, the action of $A$ on $P$ in a quantum principal bundle is determined locally by the action of the quantum group on itself or the space of polynomial functions on this group, which is essentially its dual [79].

The polynomial function space for a group such as $\mathrm{SU}(\mathrm{N})$ is generated by the set of coordinate functions $u_{j}^{i}: g \rightarrow g_{j}^{i}$, where $g_{j}^{i}$ is the $(\mathrm{i}, \mathrm{j})$ th matrix element of $g$ in the fundamental representation. Similarly, for a quantum group $\mathcal{U}$, its dual $A$ is generated by the non-commutative coordinate functions $u_{j}^{i}: \mathcal{U} \rightarrow \mathbb{C}$ and the co-product is defined to be $\Delta u_{j}^{i}=u_{k}^{i} \otimes u_{j}^{k}$. This suggests that one can choose the operation of left multiplication to be a map from $A$ to $A$

$$
\begin{gathered}
L_{g}: u_{j}^{i} \rightarrow u_{k}^{i}\left\langle u_{j}^{k}, g\right\rangle \\
L_{g}=(i d \otimes|g\rangle) \circ \Delta
\end{gathered}
$$

and the operation of right multiplication is a map from $A$ to $A$ defined by

$$
\begin{gathered}
R_{g}: u_{j}^{i} \rightarrow\left\langle u_{k}^{i}, g\right\rangle u_{j}^{k} \\
R_{g}=(|g\rangle \otimes i d) \circ \Delta
\end{gathered}
$$

where $\langle$,$\rangle represents the inner product between elements of the quantum group \mathcal{U}$ and its dually paired Hopf algebra $A$, so that $\left\langle u_{j}^{i}, g\right\rangle=g_{j}^{i} \in \mathbb{C}$. Thus, $L_{g} u_{j}^{i}=g_{j}^{k} u_{k}^{i}$ implying the following rule for left multiplication

$$
L_{g} L_{\tilde{g}} u_{j}^{i}=g_{l}^{k} \tilde{g}_{j}^{l} u_{k}^{i}=L_{g \tilde{g}} u_{j}^{i}
$$

Similarly, for right multiplication, $R_{g} R_{\tilde{g}}=R_{\tilde{g} g}$. Thus, the noncommutative function spaces, $A=\mathbb{C}\left\langle u_{j}^{i}\right\rangle /\left(R_{12} u_{1} u_{2}-u_{2} u_{1} R_{12}\right)$ are dually paired to the quantum groups

$\mathcal{U}=\frac{\mathbb{C}\left\langle l_{j}^{+i}, l_{j}^{-i}\right\rangle}{\left\{\begin{array}{l}\left(R_{12} l_{2}^{ \pm} l_{1}^{ \pm}-l_{1}^{ \pm} l_{2}^{ \pm} R_{12}\right) \\ \left(R_{12} l_{2}^{+} l_{1}^{-}-l_{1}^{-} l_{2}^{+} R_{12}\right)\end{array}\right.}$, where the $l_{j}^{ \pm i}$ are the generators of the universal enveloping algebra of the quantum group in the Chevalley basis [80]. The inner products satisfy

$$
\begin{aligned}
\left\langle u_{j}^{i}, l_{l}^{+k}\right\rangle & =R_{j l}^{i k} \quad\left\langle u_{j}^{i}, l_{l}^{-k}\right\rangle=R_{l j}^{-1}{ }_{l j}^{k i} \\
\langle a b, c\rangle & =\left\langle a \otimes b, c_{(1)} \otimes c_{(2)}\right\rangle \\
\langle a, c d\rangle & =\left\langle a_{(1)} \otimes a_{(2)}, c \otimes d\right\rangle
\end{aligned}
$$

The adjoint action on a Hopf algebra is taken to be a map $A d_{R}: A \rightarrow A \otimes A$,

$A d_{R}(a)=\sum a_{(2)} \otimes\left(S a_{(1)}\right) a_{(3)}, a \in A$. An adjoint action can also be defined as a map from $A$ to $A$

$$
\begin{aligned}
A d_{S(g)} a & =\left\langle a_{(1)}, g_{(1)}\right\rangle a_{(2)}\left\langle a_{(3)}, S\left(g_{(2)}\right)\right\rangle=R_{g}\left(a_{(1)}\left\langle a_{(1)}, S\left(g_{(1)}\right)\right\rangle\right)=R_{g} L_{g^{-1}} \\
g & \in \mathcal{U}, a \in A
\end{aligned}
$$

Given multiplication in the quantum group $\mathcal{U}$, it follows that if one considers a left translation from $y$ to $y^{\prime}=g \cdot y$,

$$
R_{y *} R_{g *}=R_{y^{\prime} *}=L_{y^{\prime} *} A d_{S\left(y^{\prime}\right)}
$$

for tangent mappings [81][82] induced by transformations from the Hopf algebra $A$ to $A$, whereas a right translation from $y$ to $y^{\prime}=y \cdot g$ implies $L_{y *} L_{g *}=L_{y^{\prime} *}$. It follows that, for a specific 
choice of parallelism, such as that defined by left multiplication, on the standard fibre in the quantum principal bundle, transformations in the structure group should only be defined using right translations rather than left translations on the Hopf algebra. Moreover, if the connection forms are required to take values on the base space subalgebra $B$, then the group of gauge transformations is an automorphism group acting on the total space $P$, which preserves the base space $B$. Previous considerations of the fibre coordinate dependence of the connection form transformations imply that it is this group which must necessarily be used in the definition of gauge transformations in the quantum principal bundle [78]. In the classical limit, for principal bundles, the automorphism group, the structure group and the standard fibre all coincide.

Specifically, if $\beta: A \rightarrow \Gamma_{B}$ is a linear map such that $\beta(1)=0$,

$$
\omega(a)=\sum \Phi^{-1}\left(a_{(1)}\right) j\left(\beta\left(a_{(2)}\right)\right) \Phi\left(a_{(3)}\right)+\sum \Phi^{-1}\left(a_{(1)} d \Phi\left(a_{(2)}\right)\right)
$$

is a connection one-form in the principal bundle $P(B, A, \Phi)$ having a trivialization $\Phi: A \rightarrow P$ [78]. Given two linear maps $f_{1}$ and $f_{2}$ on the quantum group $\mathcal{U}$ from $A$ to $B$, the convolution is $g=f_{1} * f_{2}$, where $g(a)=\sum f_{1}\left(a_{(1)}\right) f_{2}\left(a_{(2)}\right)$. If $\gamma: A \rightarrow B$ is a convolution invertible map such that $\gamma(1)=1$, then the connection form (C7) transforms as

$$
\omega^{\gamma}=\left(\Phi^{\gamma}\right)^{-1} * j(\beta) * \Phi^{\gamma}+\left(\Phi^{\gamma}\right)^{-1} * d \Phi^{\gamma}
$$

under a change of trivialization $\Phi \rightarrow \Phi^{\gamma}[78]$.

The presence of an ordinary classical group in a quantum principal bundle is consistent with a reconstruction theorem for these bundles [83]. Although the base space and total space are quantum spaces, and the structure group can selected initially to be a quantum group, the quantum bundle admits then admits sections only if it is trivial. The classification of these bundles is then reduced to the category of bundles with a standard Lie group as the structure group. 


\section{REFERENCES}

[1] E. Martinec, 'Conformal Field theory on a (Super)Riemann Surface', Phys. Lett. B171 (1986) 189-194

[2] R. Kallosh and A. Morosov, 'On Vanishing of Multiloop Contributions to 0,1,2,3 Point Functions in Green-Schwarz Formalism for Heterotic String', Phys. Lett. B207 (1988) 164-168; A. Restuccia and J. G. Taylor, 'Light Cone Gauge Analysis of Superstrings', Phys. Rep. 174 (1989) 283-407

[3] N. Berkovits, 'Finiteness and Unitarity of Lorentz Covariant Green-Schwarz Superstring Amplitudes', Nucl. Phys. B408 (1989) 43-61

[4] S. Davis, 'Summability of Superstring Theory', Phys. Lett. B432 (1998) 317-325

[5] B. R. Greene, K. H. Kirklin, P. J. Miron and G. G. Ross, 'Superstring Models with SU(5) and SO(10) Unifying Groups', Nucl. Phys. B292 (1987) 606-652

[6] P. S. Aspinwall, B. R. Greene, K. H. Kirklin and P. J. Miron, 'Searching for Three Generation Calabi-Yau Manifolds', Nucl. Phys. B294 (1987) 193-222

[7] S. W. Hawking, 'The path-integral approach to quantum gravity' in General Relativity: An Einstein Centenary Survey, ed. by S. W. Hawking and W. Israel (Cambridge: Cambridge University Press, 1979) 746-789

[8] E. Witten, 'Non-Commutative Geometry and String Field Theory', Nucl. Phys. B268 (1986) 253-294

[9] T. Banks and E. Martinec, 'The Renormalization Group and String Theory', Nucl. Phys. B294 (1987) 733-746

[10] A. Connes, 'The Action Functional in Noncommutative Geometry', Commun. Math. Phys. 117 (1988) 673-683

[11] A. Le Clair and C. Vafa, 'Quantum Affine Symmetry as Generalized Supersymmetry', Nucl. Phys. B401 (1993) 413-454

[12] S. Doplicher and J. E. Roberts, 'Why there is a field algebra with a compact gauge group describing the superselection structure in particle physics', Commun. Math. Phys. 131 (1990) $51-107$

[13] H. E. Rauch, Ann. Math. Ser. $2 \underline{54}$ (1951) 38-55

[14] D. Gromoll, 'Differenzierbare Strukturen und Metriken postiver Kreummeng auf Spharen', Math. Ann. 164 (1966) 353-371

[15] R. Geroch,'Spinor Structure of Space-times in General Relativity.I', J. Math. Phys. $\underline{9}$ (1968) $1739-1744$

[16] H. Gluck, F. Warner and C. T. Yang, 'Division algebras, fibrations of spheres by great spheres and the topological determination of space by the gross behavious of its geodesics', Duke Math. J., Vol. 50 No. 4 (1983) 1041-1076

[17] S. Davis, 'A Constraint on the Geometry of Yang-Mills Theories', J. Geometry and Physics, 4 (1987) 405-415; ICTP High-Energy Physics Seminar (1986) 
[18] A. Pais, 'Remark on the Algebra of Interactions', Phys. Rev. Lett. $\underline{7}$ (1961) 291-293

[19] M. Gunaydin and F. Gursey, 'Quark Structure and Octonions', J. Math. Phys. 14 (1973) $1651-1667$

[20] M. Gunaydin, 'Octonionic Hilbert Spaces, The Poincare Group and SU(3)', J. Math. Phys. 17 (1976) 1875-1883

[21] F. Gursey, Exceptional Groups and Elementary Particles, Lect. Notes Phys. 50 (1976) 225233

[22] M. Blencowe and M. Duff, 'Supermembranes and the Signature of Space-Time', Nucl. Phys. $\underline{B 310}$ (1988) 387-404

[23] M. Kervaire, 'Non-parallelizability of the n-sphere for $n>7$ ' Proc. Nat. Acad. Sci. USA (1958) 280-283

[24] J. F. Adams, 'Vector fields on Spheres' Annals of Math. $\underline{75}$ (1962) 602-632

[25] J. Milnor and J. D. Stasheff, Characteristic Classes, Annals of mathematics studies, no. 76 (Princeton: Princeton University Press, 1974)

[26] D. Husemoller, Fibre Bundles (New York: Springer-Verlag, 1966)

[27] W. Nahm (1978) 'An Octonionic Generalization of Yang-Mills', CERN preprint TH-2489

[28] G. Domokos, S. Kosevi-Domokos 'Gauge Fields on Coset Spaces' Il Nuovo Cimento $\underline{44 A}$ (1978) 318-329

[29] R. Dundarer, F. Gursey and C.-H.Tze, 'Selfduality and Octonionic Analyticity of $S^{7}$-Valued Antisymmetric Fields in Eight Dimensions' Nucl. Phys. B B266 (1985) 440-450

[30] M. Chaichian and N. F. Nelipa, Introduction to Gauge Field Theories (Berlin: Springer-Verlag, 1984)

[31] C. C. Lassig and G. C. Joshi, 'An Octonionic Gauge Theory', Chaos, Solitons \& Fractals, Vol. 7, No. 5 (1996) 769-775

[32] A. Ritz and G. C. Joshi, 'A Non-associative Deformation of Yang-Mills Gauge Theory' University of Melbourne preprint, UM-P-95/69, RCHEP-95/18

[33] S. Okubo, Introduction to Octonion and Other Non-Associative Algebras in Physics, Montroll Memorial Lecture Series in Mathematical Physics, Vol. 2 (Cambridge: Cambridge University Press, 1995)

[34] G. M. Dixon, 'Derivation of the Standard Model', Il Nuovo Cimento $105 B$ (1990) 349-364

G. M. Dixon, Division Algebras: Octonions, Quaternions, Complex Numbers and the Algebraic Design of Physics (Dordrecht: Kluwer Academic Publishers, 1994)

[35] Y. A. Drozd and V. V. Kirichenko, Finite dimensional algebras (Berlin: Springer-Verlag, 1994)

[36] C. A. Manogue and T. Dray, 'Dimensional Reduction', Mod. Phys. Lett. A14 (1999) 99-104 
[37] L. V. Lipagina, 'On the Algebra of Invariant Affinor Structures on the Sphere $S^{5}$,' Russian Mathematics (Iz. VUZ) Vol. 41, No. 9 (1997)

[38] K. Nomizu, 'Invariant affine connections on homogeneous spaces', Amer. J. Math. $\underline{76}$ (1954) 33-65

[39] A. Elduque and H. C. Myung, 'Color Algebras and Affine Connections', J. Algebra 149 (1992) 234-261

[40] A. Elduque and H. C. Myung, 'The reductive pair $\left(B_{3}, G_{2}\right)$ and affine connections on $S^{7}$, J. Pure and Applied Algebra $\underline{86}$ (1993) 155-171

[41] G. W. Schwarz, 'Invariant Theory of $G_{2}$ and $\operatorname{Spin}(7)$ ', Comment. Math. Helvetica $\underline{63}$ (1988) 624-663

[42] A. V. Iltyakov, 'Trace Polynomials and Invariant Theory', Geometriae Dedicata $\underline{58}$ (1995) 327333

[43] G. Domokos, S. Kosevi-Domokos, 'The Algebra of Color', J. Math. Phys. 19 (1978) 1477-1481

[44] T. D. Lee, Particle Physics and Introduction to Field Theory, Contemporary Concepts in Physics, Vol. 1 (Chur: Harwood, 1981)

[45] N. Manton, 'Generalized Dimensional Reduction of Supergravity' Annals of Physics, 167 (1986) 328-353

[46] E. Witten, 'Dimensional Reduction of Superstring Models', Phys. Lett. B155 (1985) 151-154

[47] P. Forgacs and N. Manton, Space-Time Symmetries in Gauge Theories, Commun. Math. Phys. $\underline{72}(1980)$ 15-35

[48] N. Manton, 'A New Six-Dimensional Approach to the Weinberg-Salam Model', Nucl. Phys. $\underline{B 158}$ (1979) 141-153

[49] R. Jackiw, Diverse Topics in Theoretical and Mathematical Physics (Singapore: World Scientific Publishing Company, 1995)

[50] C. Pope and N. Warner, 'An SU(4) Invariant Compactification of D=11 Supergravity on a Stretched Seven Sphere', Phys. Lett. B150 (1985) 352-356

[51] B. de Wit and H. Nicolai, 'The Embedding of Gauged N=8 Supergravity into D=11 Supergravity', Nucl. Phys. B255 (1985) 29-62

[52] L. N. Chang and F. Mansouri, Phys. Rev. $\underline{D 17}$ (1978) 3168-3178

[53] S. Coleman, J. Wess and B. Zumino, 'Structure of Phenomenological Lagrangians I', Phys. Rev. $\underline{D 177}(5)(1969) 2239-2247$

[54] Kuang-Chao Chou, Tung-Sheng Tu and Tu-Nan Yean, 'The Pure Gauge Fields of a Coset Space', Vol.XXII No. 1, Scientia Sinica (1979) 37-52

[55] N. Berkovits, 'A Supertwistor Description of the Massless Superparticle in TenDimensional Superspace', Phys. Lett. B247 (1990) 45-49

[56] M. Cederwall, 'An Extension of the Twistor Concept to String Theory', Phys. Lett. $\underline{B 266}$ (1989) 45-47 
[57] C. A. Manogue and A. Sudbery, 'General Solution of Covariant Equations of Motion', Phys. Rev. D40 (1989) 4073-4077

[58] A. Sudbery, 'Division Algebras, (pseudo)orthogonal groups and spinors', J. Phys. A 17 (1984) 939-955

[59] K.-W. Chung and A. Sudbery, 'Octonions and the Lorentz and Conformal Groups of Ten-Dimensional Space-Time', Phys. Lett. B198 (1987) 161-164

[60] M. Cederwall, 'Introduction to Division Algebras, Sphere Algebras and Twistors', Talk presented at the Theoretical Physics Network Meeting at NORDITA, Copenhagen, September, 1993

[61] M. Cederwall, 'Octonionic Particles and the $S^{7}$ Symmetry', J. Math. Phys. 33 (1992) 388-393

[62] M. Cederwall and C. R. Preitschopf, ' $S^{7}$ and $\hat{S}^{7}$, Commun. Math. Phys. 167 (1995) 373-393

[63] N. Berkovits, 'A Supertwistor Description of the Massless Superparticle in TenDimensional Superspace', Phys. Lett. B247 (1990) 45-49

[64] L. Brink, M. Cederwall and C. R. Preitschopf, ' $N=8$ Superconformal Algebra and the Superstring', Phys. Lett. B311 (1993) 76-82

[65] E. Witten, 'Twistor-Like Transform in Ten Dimensions', Nucl. Phys. B266 (1986) 245-264

[66] G. Moreno, 'The zero divisors of the Cayley-Dickson algebras over the real numbers' (1997) q-alg/9710013

[67] B. Eckmann, 'Gruppentheoretischer Beweis des Satzes von Hurwitz-Radon ueber die Komposition quadratisher Formen', Comment. Math. Helv. 15 (1943) 358-366

[68] N. N. Vakhania and N. P. Kandelaki, 'A Generalization of the Hurwitz-Radon-Eckmann Theorem and Orthogonal Random Vectors', Soviet Math. Dokl. 36(2)(1988) 245-246

[69] N. N. Vakhania, 'Orthogonal Random Vectors in Hilbert Spaces and a Related Problem of Linear Algebra' in the Proceedings of the 3rd Symposium on Probablility and Stochastic Processes held at Hermosillo, Mexico. Sociedad Matematica Mexicana: Aportaciones Mat., Notas Invest. 11 13-30 (1994)

[70] M. Koca and N. Ozdes, 'Division algebras with integral elements', J. Phys. A 22 (1989) 14691493

[71] A. N. Schellekens, Phys. Lett. $\underline{B 199}$ (1987) 427-431

[72] A. A. Albert, 'The structure of any algebra which is a direct product of rational generalized quaternion division algebras', Ann. Math. $\underline{30}$ (1929) 621-625

[73] E. Dieterich, 'Real quadratic division algebras', Comm. Alg. 28 (2)(2000) 941-947

[74] B. A. Dobrescu and E. Poppitz, Phys. Rev. Lett. $\underline{87}$ (2001) 031801

[75] P. Libermann, 'Parallelismes', J. Diff. Geom. $\underline{8}$ (1973) 511-537

[76] H. Rund, 'Variational Principles for Field Variables that are Subject to Group Actions' Algebraic and Differential Topology - Global Differential Geometry, ed. by G. M . Ras- 
sias (Leipzig: B. G. Teubner, 1984)224-251

[77] J. Wolf, 'On the Geometry and Classification of Absolute Parallelisms I', J. Diff. Geom. $\underline{6}$ (1972) 317-342; 'On the Geometry and Classification of Absolute Parallelisms II' $\underline{7}$ (1972) 19-44

[78] T. Brzezinski and S. Majid, 'Quantum Group Gauge Theory in Quantum Spaces', Commun. Math. Phys. 157 (1993) 591-638

[79] S. Majid, Foundations of Quantum Group Theory (Cambridge: Cambridge University Press, 1994)

[80] S. Majid, 'Quasitriangular Hopf Algebras and Yang-Baxter Equations', Int. J. Mod. Phys. $\underline{A 5}$ (1990) 1-91

[81] P. Schupp, 'Cartan Calculus:Differential Geoemetry for Quantum Groups', Enrico Fermi Summer School on Quantum Groups,

Lake Como 1994, Quantum Groups and Their Applications

in Physics, ed. by L. Castellani and J. Wess, IOS Press, 1996

[82] P. Aschieri and P. Schupp, 'Vector Fields on Quantum Groups', Int. J. Mod. Phys. $\underline{A 11}$ (1996) $1077-1100$

[83] R. J. Budzynski and W. Kondracki, 'Quantum Principal Fibre Bundles: Topological Aspects, Rep. Math. Phys. 37 (1996) 367-385 